\title{
Pragmatic Audiovisual Preservation
}

\section{Ashley Blewer}

\section{DPC Technology Watch}

\section{Report}

October 2020

DigitalPreservationCoalition 
(C) Digital Preservation Coalition 2020 and Ashley Blewer 2020

ISSN: 2048-7916

DOI: http://doi.org/10.7207

All rights reserved. No part of this publication may be reproduced, stored in a retrieval system, or transmitted, in any form or by any means, without prior permission in writing from the publisher. The moral rights of the author have been asserted.

First published in Great Britain in 2020 by the Digital Preservation Coalition.

V1.1 Minor corrections added in September 2021. 


\section{Foreword}

The Digital Preservation Coalition (DPC) is an advocate and catalyst for digital preservation, ensuring our members can deliver resilient long-term access to digital content and services. It is a not-forprofit membership organization whose primary objective is to raise awareness of the importance of the preservation of digital material and the attendant strategic, cultural and technological issues. It supports its members through knowledge exchange, capacity building, assurance, advocacy and partnership. The DPC's vision is to make our digital memory accessible tomorrow. The DPC Technology Watch Reports identify, delineate, monitor and address topics that have a major bearing on ensuring our collected digital memory will be for the future.

They provide an advanced introduction in order to support those charged with ensuring a robust digital memory, and they are of general interest to a wide and international audience with interests in computing, information management, collections management and technology. The reports are commissioned after consultation among DPC members about shared priorities and challenges; they are commissioned from experts; and they are thoroughly scrutinized by peers before being released. The authors are asked to provide reports that are informed, current, concise and balanced; that lower the barriers to participation in digital preservation; and that are of wide utility. The reports are a distinctive and lasting contribution to the dissemination of good practice in digital preservation.

This report is published by the DPC with the support of the Research \& Practice Subcommittee who provide editorial oversight. It was written by Ashley Blewer, archivist, developer and technologist, specializing in video preservation, digital repository management, infrastructure/community building, computer-to-human interpretation, and teaching technical concepts.

\section{Acknowledgements}

I would like to deeply thank my writing group teammates of eight years and counting, Marleigh Chiles and Travis L. Wagner, for giving me the strength and motivation to continue onward and upward, no matter what. Thank you to all open-source audiovisual preservation software creators, but especially Dave Rice for his generosity and commitment to open and accessible audiovisual preservation.

Further thanks to Linda Tadic, for a number of minor additions, corrections and clarifications. 


\section{Contents}

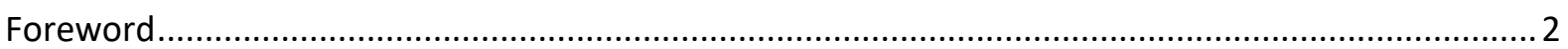

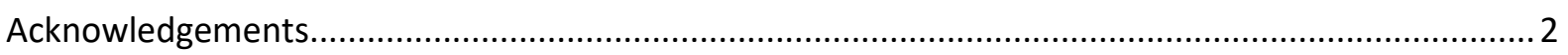

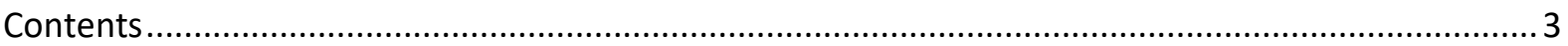

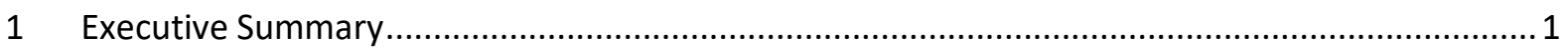

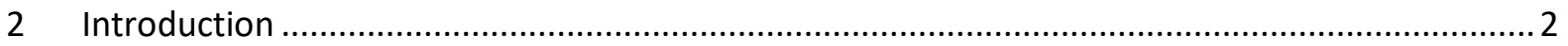

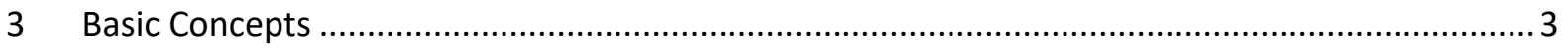

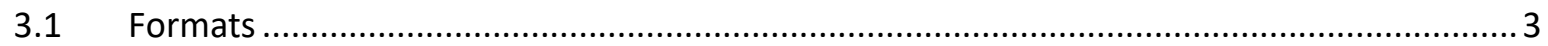

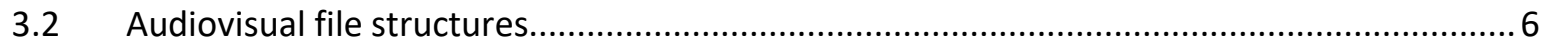

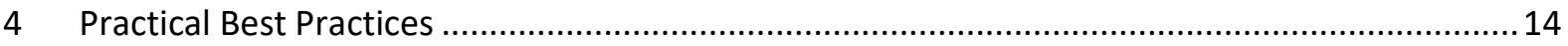

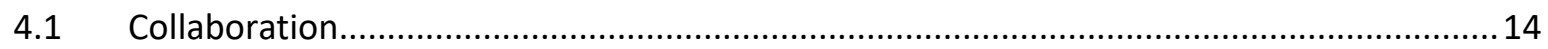

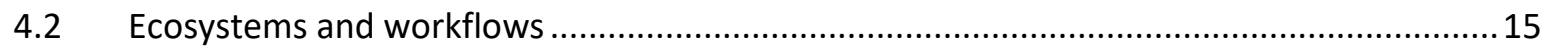

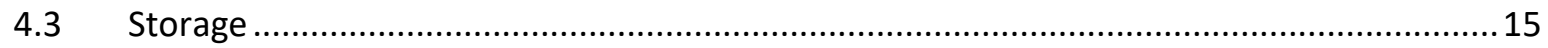

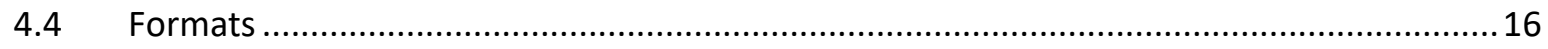

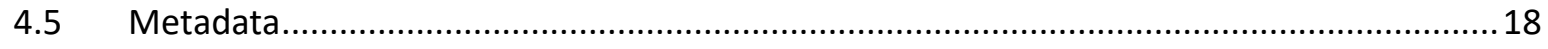

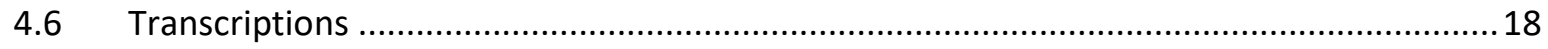

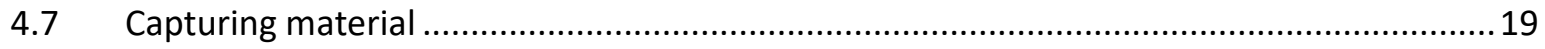

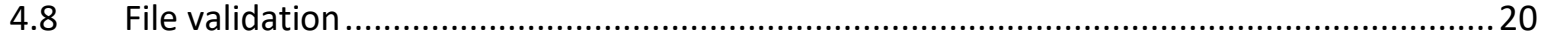

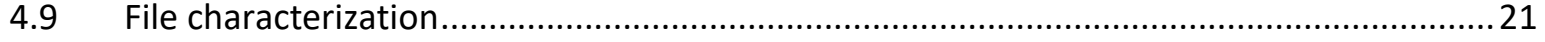

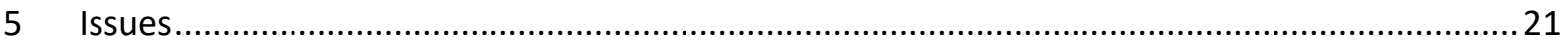

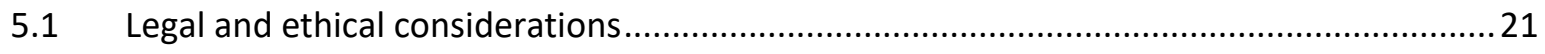

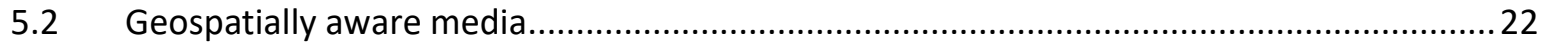

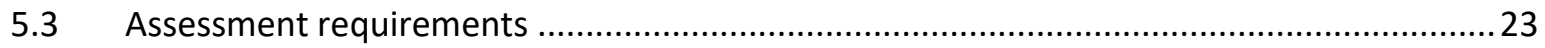

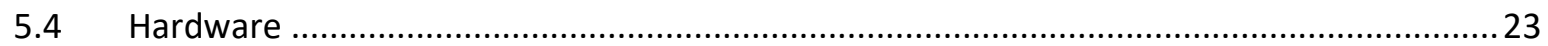

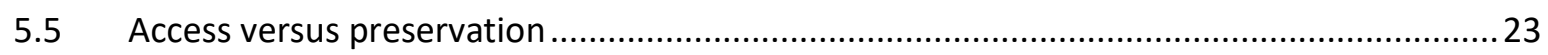

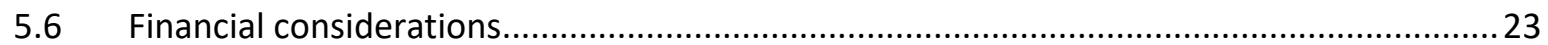

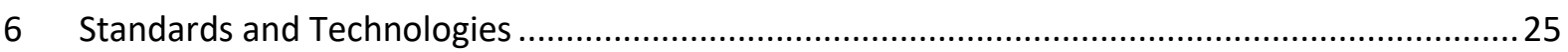

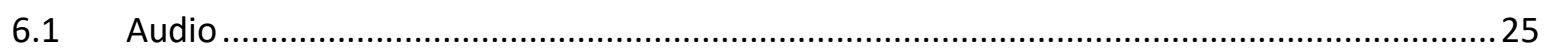

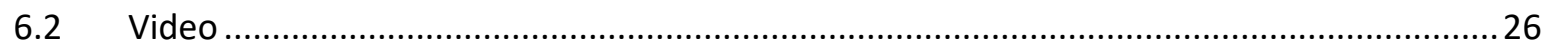

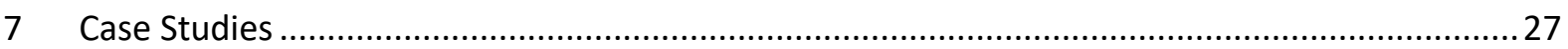

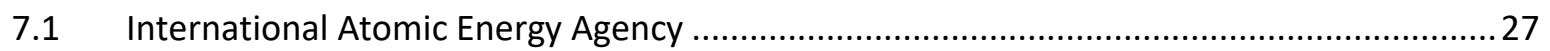

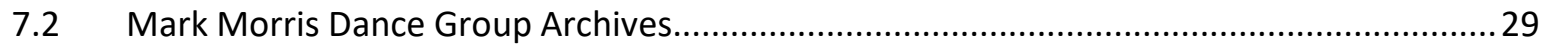

7.3 Borthwick Institute for Archives at the University of York .................................................. 31

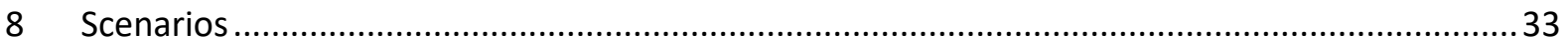

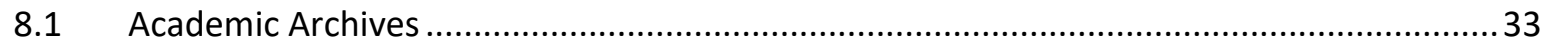

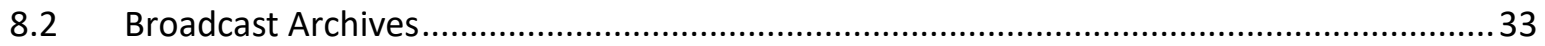




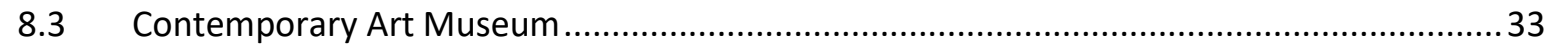

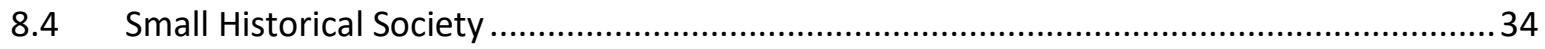

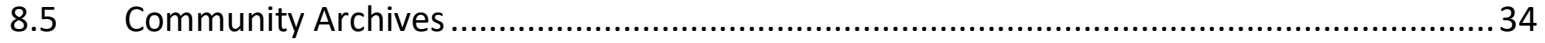

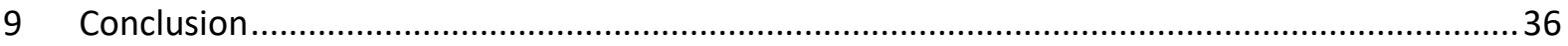

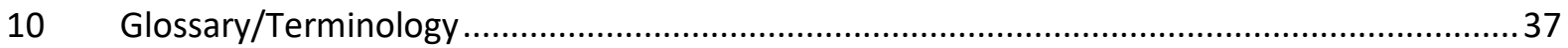

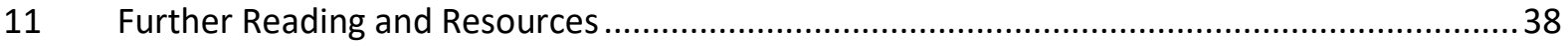

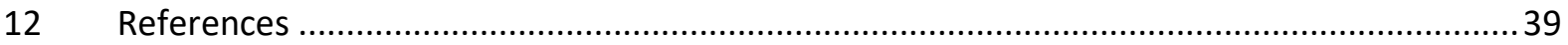




\section{Executive Summary}

This report provides pragmatic advice on the processing of audiovisual material with the intent of long-term preservation and access. It delivers practical and approachable guidelines on audio and video preservation for organizations of all sizes.

Pragmatic Audiovisual Preservation sits alongside the Digital Preservation Coalition Technology Watch Report, Preserving Moving Pictures and Sound (Wright, 2012). It builds on this document rather than superseding it. In particular Preserving Moving Pictures and Sound should be consulted for information about the digitization of analogue materials, determining and maintaining audiovisual signal quality, and audio and video standards.

This report covers the fundamental concepts of existing audio and video formats, from analogue to digital, and including hybrid formats. It focuses on the technical properties of digital videos, highlighting the most significant aspects of each that should be considered when working with audiovisual materials. This includes containers, codecs, colour, bit depth, frames, samples, pixels, and timecodes.

The report moves from large and abstract concepts to specific details, explaining and describing good practice. Topics covered include collaboration, ecosystems and workflows, storage, formats, metadata, transcriptions, capturing material, file validation, and file characterization.

There are multiple issues specific to audiovisual preservation that preservationists should be aware of when launching projects working with this type of material. The issues covered within this report are rights and copyright, capture of ephemeral material, format confusion, storage, time, assessment requirements, hardware, financial issues, and the tension between preservation and access.

Also included within this report are a range of case studies from real institutions who are tackling this preservation challenge, and hypothetical scenarios that focus on the different ways that an organization may address the handling of audiovisual material, depending on their priorities, time and finances. These case studies and scenarios may be used to provide insight into some of the ways organizations might best preserve their material depending on the context in which they are operating. 


\section{Introduction}

The preservation of audiovisual materials remains a challenging topic. Moving beyond the complexity of audiovisual materials and the urgency around digitization, concepts related to significant technical characteristics and the impact each has on digital audiovisual files can be daunting. These challenges are emphasized by the emergence of advanced video formats such as Virtual Reality, Augmented Reality, and $360^{\circ}$ video.

This report therefore aims to provide easily digestible guidance for practitioners with a basic knowledge of digital preservation concepts and archival practices but without expertise in audiovisual materials. It does this by providing a foundation in the basics of audiovisual preservation and practical steps on taking forward the preservation of these materials in typical organisational settings. It intentionally avoids providing high levels of detail around some of the specifics of audiovisual formats and the particular issues that can arise in their preservation - but a comprehensive further reading list is provided at the end of the report for reference purposes. It makes no assumptions about the size, capability, or infrastructure of the preserving organisation and aims to provide guidance of equal relevance to those with limited resources.

It is important to note that 'best practice' is often an unrealistic and inappropriate aim. Institutions must perform preservation actions to their specific standards and goals, within the constraints of time and resourcing and in order to meet particular needs. This report therefore follows the broader DPC principle of delivering realistic and practical guidance that might better be termed 'good practice'. 


\section{Basic Concepts}

This section focuses on developing a foundation of preservation concepts as they relate to audiovisual materials. It is separated into two categories: Formats and Audiovisual file structures. The first covers analogue and digital formats and the preservation challenges surrounding each. The second seeks to comprehensively cover some of the essential technical components of digital audiovisual files.

The Formats section covers the basic concepts of working with audiovisual materials. These materials are divided into the categories of analogue, digital, or hybrid materials. The analogue section will provide an overview of the categories of imprinted media, film, and magnetic media, as other literature covers this topic in depth. The digital section will be very succinct, as the following Audiovisual file structures section covers the technical details of file-based digital objects. The last section focuses on Hybrid media and will include some examples in which audiovisual material can be simultaneously analogue and digital, such as when digital content is on an analogue carrier.

The Audiovisual file structures section covers fundamental concepts related to how audio and video work at a technical level, looking at the essential components that make up an audiovisual file, how to use this knowledge, and why it is significant when preserving this material. The section will cover the concepts of containers, codecs, colour, bit depth, frames, pixels and timecodes.

\subsection{Formats}

Audiovisual materials can be broken down into two largely distinct categories, analogue and digital. This report covers an additional third category, in which the first two are consolidated into specific formats, and thus issues from the analogue and digital realms of sound and moving image materials must be considered throughout the archival lifecycle.

Simplifying types of audiovisual material into these categories leaves out a lot of nuance when it comes to fully understanding the depth and breadth of archival objects. However, this method was chosen as a way to distinguish audiovisual formats found within existing archival collections, and a basic assessment will need to be performed before tackling the management of long-term preservation of specific objects. Because of this, the audiovisual material is considered through format carriers rather than summarized by how the information is stored.

While it is important to be aware of the differences and nuances between audiovisual materials, the following concepts will focus only briefly on different formats. Details are limited to what is necessary for the context within which to understand how analogue materials come into existence before being transformed into a digital format for preservation and/or access. Additional details on analogue audiovisual materials and their digitization process can be found in the first version of this publication (Wright, 2012) and other well-established resources. See the Further Reading section of this report for more information beyond what is covered here.

\subsubsection{Analogue}

Analogue material can be thought of as any moving image or sound content that was created and stored on non-digital material. This can be summarized into three categories: imprinted media, film and magnetic media.

\subsubsection{Imprinted media}

Imprinted media is any material that has been engraved onto the outer surface of a physical material and can be reproduced when played on a relevant device. Examples of imprinted media include vinyl records, aluminium discs, and wax cylinder sound recordings. These media rely on equipment that 
can run along grooves embedded into a physical object and produce a sound recording. Imprinted media is particularly fragile and must be handled carefully as part of the digitization process, and stored with care in climate-controlled environments.

\subsubsection{Film}

Film consists of an emulsion process that creates an image on photosensitive plastic material. This plastic can be cellulose nitrate, poly-acetate, and other formats. Here are some of the most common film formats:

- Nitrate: Also referred to as cellulose nitrate or nitrocellulose, this format was used from the advent of the film age in the 1880s up until the 1950s. Nitrate film catches fire very easily and there may be local regulations that require the film to be stored and transported carefully.

- Acetate (diacetate and triacetate): A film base created to replace the more dangerous nitrate, and used in major motion picture film as well as small-gauge film. When ageing in poor conditions, it can suffer from 'vinegar syndrome', named after its smell.

- Polyester: The most modern film base, and often used when creating preservation-grade copies of films on film due to its stability.

Moving images are displayed per frame, with frames moving at a rate of 24 frames per second in most cases, but this may differ depending on the format. This standardization of 24 frames per second was introduced with the advent of sound waves printed on film. This synced sound forced previously inconsistent recording and playback rates to become standardized. See 3.2.5 Frames for more details on frame rates.

\subsubsection{Magnetic media}

Magnetic media encompasses all forms of audiovisual material that stores data on a magnetized plastic medium. This format stores different data patterns that are replayed through the interpretation of a magnetic strip of plastic. Magnetic media is a tricky and fragile format, and experts estimate that it is essential to move archival material from this medium by 2030-2035 (Casey, 2015). After that point, it is expected that there will no longer be enough functional decks to replay material and the existing material will have degraded too severely to be played back.

\subsubsection{Hybrid}

Keep in mind that analogue and digital formats are not mutually exclusive, and combinations of these materials can be one format. For example, a $16 \mathrm{~mm}$ moving picture film with a magnetic media audio track combines both film and magnetic media into one difficult hybrid format.

Hybrid media consists of any audiovisual material that is a combination of digital data and a physical medium. These materials have all of the issues that both analogue and digital materials have. Hybrid materials are at the greatest preservation risk, given their various dependencies: keeping the physical material in good shape and without wear, keeping the equipment functional for replay, and sometimes the software required to access the material. Hybrid audiovisual media should be considered to be in the highest risk category for migration.

Below are short summaries of two common hybrid format categories. Keep in mind that there are many more irregular formats, and additional research will need to be done to understand and care for more rare and obsolete carriers and machinery. 


\subsubsection{Optical media}

Optical media refers to digital material on physical discs that are read by laser-based playback devices. Examples of this format include CD-ROMs, music-based CDs, Blu-ray, and MiniDisc. Generally speaking, optical media do not have a long 'shelf life'. Mass produced CD-ROMs and Bluray discs will typically not contain the original version of content. Discs that were burnt individually with commodity optical drives (such as CD-R, DVD-R or BluRay BD-R) may however hold unique or original data. MiniDisc players had an ability to record directly onto MiniDiscs, and may have been used to capture interviews or live recordings.

DVDs have been used to store archival material such as digitized home movies, particularly from commercial, non-archival vendors. Anything stored on non-archival optical media should be considered to be in the highest risk category for migration, particularly material upwards of 10 years old, and any material not stored carefully in a consistently cool location with low light. Light is one of the most damaging elements to optical media formats. Optical media was a popular format for writing material to, and sharing copies of, media. More information can be found in An Introduction to Optical Media Preservation published in the Code4lib journal (Duryee, 2014).

\subsubsection{DV}

DV is a family of file formats featuring digital content written to magnetic media tape and stored in plastic carriers, popular from the late 1980 s to early 2010 s for use in video production, broadcast media, and consumer use. DV is short for Digital Video, which can be confusing because that also describes a concept (see next section) or other hybrid formats like Digital Betacam. The significance of the DV family, particularly MiniDV, is that it is very likely to contain original material not available or copied to another source, particularly in the context of amateur-produced media like home movies, or footage taken during live events. More information can be found through the DVRescue initiative, which contains knowledge and tools for preserving this format (MIPoPS, 2020). Note that there are two types of magnetic tape: Metal Evaporated (ME) and Metal Particle (MP). ME, used by Sony, can wear when played back or paused and should be recovered as a priority.

\subsubsection{Digital}

The majority of audiovisual material created in the 21st century has been digital and stored as files. With digital formats, everything compiles down into ones and zeros, but there is still a massive amount of nuance within those binary formats.

An essential component to working with digital material is ensuring that it can be made available and accessible in the future. Content that works with machines from the near-past (within the last decade) cannot necessarily be played on today's equipment. For example, ten years ago it was standard for new computers, desktop or laptop, to come with a CD/DVD player, while today they are something you have to buy as an extension and are not considered the default.

Within the context of preservation, digital audiovisual material can be thought of in three major categories: digitized, file-based, and stream-based material. Digitized and born-digital content end up in a specific digital structure. File formats relay some information about what kind of video a certain file is by indicating its carrier container, but it only tells part of the story. The other part of audiovisual material is described by how its data is encoded.

\subsubsection{Digitized}

Digitized audiovisual material is any material that started its life as an analogue or hybrid medium and has been recreated as a digital representation of the original media. For digitized material, the 
moment of being transferred from its original analogue medium into a digital file is the largest preservation treatment decision that will be performed in its life. Preservationists have a lot of control over digitized material and have the ability to make significant decisions surrounding their conversion. See the previous report (Wright, 2012) for more details of this part of the object lifecycle.

\subsubsection{Born-digital (file-based)}

Born-digital materials are objects which came into existence as digital objects, and usually subsequently become files. This material can be one of hundreds of formats. These formats may be proprietary, limited to specific devices or operating systems, and may cause difficulty for digital preservation due to the inability of other software or systems to understand and play back the files. Extra care must be given when working with formats that are higher risk. More research can be conducted on a file format basis by looking into the 'Bit List' of Digitally Endangered Species (Digital Preservation Coalition, 2019)

\subsubsection{Born-digital (stream-based)}

Born-digital content is increasingly created on-the-fly and streamed directly onto publishing platforms such as YouTube or Instagram. This material essentially only lives as a data stream and does not end up as a file, at least not in a traditional sense. Even though there is, in theory, a primary copy of a file available in some context, this may not be available to be appropriately retrieved and handled according to modern archival practices, which are file-based and have the expectation of a single, high-quality, prepared version of the content.

\subsection{Audiovisual file structures}

This section introduces concepts essential to understanding how digital video works, and the many attributes that need to be considered when working with digital materials, especially at the point of digitization, conservation treatment, accessioning, digital-to-digital migration, or during the creation of Dissemination Information Packages (DIPs), in OAIS Reference Model parlance (CCSDS Secretariat, 2012).

To begin, it is important to understand that unlike many other digital file formats, videos are more than just a singular format. Videos have a container format with additional formats embedded inside, and each of those components must be described to understand the entire video file - not just the file extension.

\subsubsection{Containers}

Audiovisual material consists of multiple streams of data: a common structure is one video stream and one audio stream. There can be other streams, like additional audio streams for different 'dubs' of a video with both English and French audio tracks, or embedded subtitles to handle both of those languages. These streams have to be 'wrapped up' together into one format, and that is the file's container.

Containers may also be referred to interchangeably as 'formats' or 'wrappers'.. But they do more than just hold the contents of a file together. They are responsible for identifying the data, knowing how much of the data there is, understanding what kind of data streams are present and information about them, storing time data, self-identification data, and metadata about itself and its contents.

Containers also establish the appropriate file extension for the file (like .mov or .mp3), which is why they are often thought of as the audio or video format without consideration for its internal encoded 
data streams. The container is just part of what determines the essence of digital audiovisual material.

A video file can only have one container but can (and probably does) contain multiple encoded streams of data. Sometimes containers and their encoding algorithms have the same name. This is because, in those cases, the muxer (the tool that creates the container) and codec (the tool that encodes the audio or video stream) must be tied together in order to function as an audiovisual file. For example, the Windows Media Player container (using the extension of .wmv) can only contain data encoded by the Windows Media Player codec. They are intrinsically bound in that way. Other containers, in particular ones that are open formats, are less prescriptive about their contents and can handle many different data streams.

The process of putting encoded data into a container is known as 'muxing',. short for multiplexing. This is the process of combining one or more audiovisual signals, or streams, together into one file format. As previously mentioned, for a video file this can include one or more video, audio, subtitles, or data tracks. All of the parts that make up a video have to be put together and synced together in time so that it plays back in an expected and repeatable way.

Similarly, the process of taking encoded data streams out of their storage container is called 'demuxing', a shortened form of demultiplexing.

Any type of manipulation of audiovisual material, whether for preservation or access, will very likely require demuxing and remuxing to separate the streams and then perform the transformations on them, whatever that may be. For example if a preservationist wants to change the encoding of a video stream but leave the audio as-is, they will need to demux, perform that re-encoding operation, and then remux the file to put it back into the container just as it was before, with the modified stream. Most file-editing programs will make this process appear to be seamless, but it is good to know what is going on 'under the hood'; especially when unexpected things can happen, including detrimental changes to the files, that will require further investigation.

A 'muxer' is the engine that performs the process of putting data streams back together into a single video file, and, expectedly, a 'demuxer' is an engine that takes video files apart.

\subsubsection{Encodings}

A codec encodes and decodes a data stream or a signal for transmission and storage. Just like modem (modulator-demodulator), it is in the name: codec (coder-decoder). Within the context of video, that data stream can be video or audio. This encoding can be fit for a specific purpose, such as for archival storage, for transmission, or for encryption.

Most codecs deal with this concept of compression, and they seek to compress media in a way that saves space but does not look or sound like a reduction in quality. Compression of data is a primary focus of many codecs, because they are focused on streaming media on the web in the best-looking way. As preservationists, we are less concerned with optimizing for transmission and more concerned with quality of media across time.

Codecs can use lossy or lossless compression. Compression makes files smaller but does not necessarily mean that the files will lose data, so codecs are described as being either lossless or lossy. Lossy means the original stream of data, after encoding, cannot be decoded back to its original state. Lossless compression means that the encoding process is reversible, and the data can be returned to the same state as prior to encoding/compression. 
A higher-level analogy to this is compressing a file, like a text file, into a .zip file for storage or transfer. When the file is unzipped, it is the same as it was before it was zipped. The original data has not been changed or lost in any way, but the text file inside the .zip file is smaller than the text file on its own. This is the same concept for other forms of compression, like video. This is termed lossless compression.

Codecs that offer lossless compression and are open standard formats are safe to use for preservation purposes because the data itself has a reversible condition, and the information for decoding that data and bringing it back to its original state is available.

Note that perceptually lossless (sometimes referred to as visually lossless) is not the same as being a lossless encoding, but rather a codec that asserts itself as being so good at encoding that the image cannot be identified as lossless by the human eye. Codecs that describe themselves in this way should not be used for preservation, except for circumstances in which files must be compressed and there is no alternative.

Codecs do not necessarily inform video quality, as some codecs can have profiles that modify the algorithm to compress more tightly (for smaller file size) or prioritize quality (with bigger file size), among other things.

For example, h.264 as a codec can mean wildly different things based on the profile applied when encoding. h.264 refers to the algorithm used to compress media, but that algorithm can be configured in different ways based on a standard profile (Wikipedia Advanced Video Coding: Profiles $\left.2020^{1}\right)$. h.264's Baseline Profile will be more lossy but a smaller file size, and more appropriate for mobile applications or streaming media. The High 4:4:4 Predictive Profile (Hi444PP) supports greater colour depth (up to 14 bits per sample) and as such is more suited to professional applications.

When working with digital video, a preservationist may be tempted to transcode formats from one format to another, selecting a recommended target preservation format. It is important to keep in mind that if a video file has already been compressed, or if it has been 'born compressed', there is no way to undo that damage and create a better preservation copy of the file. The original copy of the file or a losslessly compressed version of it is going to be the best quality copy. Attempting to transcode a lossy video into a preservation copy will take up a lot of space, but the quality will be just as bad, if not worse, than the original.

Here are some key phrases and definitions to remember when working with codecs:

- Uncompressed: data stream with no attempts to decrease size

- Compressed: data that has been made smaller in some way

- Losslessly compressed: data that has been made smaller in some way, but the process is reversible

- Lossy compressed: data that has been made smaller and this process cannot be undone

\subsubsection{Colour}

Colour is a component crucial to video, and replicating human vision in analogue or digital media has been a highly complex science. Colour is represented by the concept of colour spaces, which is how colour is represented within an image. Different colour spaces are used in video materials,

\footnotetext{
${ }^{1}$ Wikipedia Advanced Video Coding: Profiles https://en.wikipedia.org/wiki/Advanced Video Coding\#Profiles,
} 
depending on their source or any conversions that have occurred. Care must be taken if converting from one colour space format to another, because there are technical nuances that can create a poor-quality or inaccurate image. For a deeper look into colour, see Charles Poynton's A Technical Introduction to Digital Video, cited in the Further Reading section.

These are some of the most common colour spaces to be aware of when working in video preservation:

- $\mathrm{RGB}$

- YUV

- $\mathrm{YCbCr}$

There are many other colour spaces in use, but these are the three major ones. Colour spaces follow two different conceptual models: additive colour spaces and luma/chroma colour spaces.

\subsubsection{Additive colour spaces}

RGB is the de-facto colour space for digital spaces in general, not just video. For a colour space like $\mathrm{RGB}$, there are three essential colours and they all convene in brightness, with cumulative colour creating pure white at the centre, and dark tones at the edges. Each pixel is represented by a percentage of red, green, and blue. If all three components are at $100 \%$, the colour of the pixel will be white. If all three components are at $0 \%$, the pixel will be black.

\subsubsection{Luma/chroma colour spaces}

For a colour space like YUV and $\mathrm{YCbCr}$, the representation of colour is separated by storing data on the brightness of the pixel (the $Y$ value, representing luma) and the two other parts representing the colour (referred to as chroma). Using $\mathrm{YCbCr}$ as an example, $\mathrm{Y}$ represents the luma, and $\mathrm{Cb}$ and $\mathrm{Cr}$ represent the blue-difference and red-difference of an image.

YUV and $\mathrm{YCbCr}$ are closely related but different colour spaces. They are often used interchangeably, but experts appreciate the nuances between the two. YUV is used specifically for the analogue encoding of data, like with pre-digital broadcast television, and $\mathrm{YCbCr}$ is the technology used in digital video.

Another detail worth noting is the use of the word luma, which is not to be conflated with luminance. Luminance is the intensity of light, and luma is the perceptual brightness. When working with colour spaces, the brightness is perceived, rather than something that can be measured with a photometric camera.

\subsubsection{Bit depth}

Bit depth, when speaking about video, refers to the amount of information stored for images that appear on screen. This bit depth information is stored for each bit plane. For colour video, this bit depth covers three planes: typically Red/Green/Blue for RGB or Lightness/Blue-difference/Reddifference for YcbCr.

A generally recommended bit depth for standard definition video is 8 bit. A bit depth of 8 means that there are 256 possible colours available for a particular pixel, just like early video game systems were referred to as having ' 8 - bit colour'. Although 256 colours is not very much, it is important to remember that for video, the bit depth number is applied to all three components of the colour space. So when this concept of 8 bit (depth) with 256 possible colours is conveyed per each video plane, it means that 8 -bit video is representing a colour range of $256 \times 256 \times 256$, or $16,777,216$ shades 
of colour and lightness. Similarly, 10 bit represents a colour range of $1024 \times 1024 \times 1024$ and 16 bit represents a colour depth range of $4096 \times 4096 \times 4096$. All of these numbers add up to more colours than humans are capable of perceiving.

There are reasons to preserve content above that which a human is capable of visually perceiving, and that relates to material that may have value in being colour-graded in the future or manipulated in any way. Because of this, some archives prefer using a higher resolution when setting digitization quality standards.

\subsubsection{Frames}

Audiovisual materials are made up of frames - both in video, where frames are a series of images, and in audio, where frames are a series of samples.

In video, frames are a relatively straightforward concept, where each frame is equal to one image being displayed for a set amount of time. Each of these images is made up of rows and columns of pixels. The Resolution of the image is defined by the number of pixels across and the number of pixels vertically. For example, Full HD (or "1080p") has a resolution of 1920 pixels across by 1080 pixels vertically. Sometimes this is summarised by rounding up the horizontal resolution. For example, Full HD can be known as " $2 \mathrm{~K}$ ". The greater the number of pixels, the higher the definition of the image.

\subsubsection{Samples}

Audio files are composed of samples. Samples are values at a specific point in time. Audio is often described by its sampling rate, generally presented as samples per second. This is usually displayed in Hertz (e.g. $96 \mathrm{kHz}$ ) but could also be written out fully (e.g. 96,000).

Here are some common audio sampling rates:

- $8 \mathbf{~ k H z}$ : the general sampling rate for telephone transmission

- $\quad 44.1 \mathrm{kHz}$ : the sampling rate for audio CDs

- $48 \mathrm{kHz}$ : the standard audio sampling rate used in video equipment

- $96 \mathrm{kHz}$ : the general recommendation for preservation audio

In the context of audiovisual files, audio frames consist of all the audio samples taken during the interval of the video frame.

\subsubsection{Frame rates}

Video files also have sampling rates, but are more frequently characterized by their frame rates. Frame rates determine how fast things go. Before the advent of sound-on-film, when soundwaves would be printed alongside image frames, there was no standard for the number of frames per second that a piece of media should be set at and replayed. For film captured via hand-cranked devices, the frame rate could vary depending on how quickly or slowly the cameraperson was winding the film at the time of capture. Typically, the frame rate of silent-era film was 16 or $18 \mathrm{fps}$ (frames per second). This matters when film is digitized, because it is important to respect the original frame rate and not have film appear to be 'sped up'.

Part of the reason why bad transfers of silent film can appear to be moving faster than the natural, captured movement is that when sound on film arrived, it set frame rates to the standard of $24 \mathrm{fps}$. This was the standard for film for most of its lifetime (1920s-2000s), and only recently in the 21st century have filmmakers been experimenting with frame rates higher than this. 
Frame rate speeds have often depended on the three major standards, NTSC, PAL and SECAM. NTSC (National Television Standards Committee) was the standard broadcast format in the United States with a frame rate for video of $29.97 \mathrm{fps}$. PAL (Phase Alternating Line) was the standard broadcast format in Europe and Asia with a frame rate of $25 \mathrm{fps}$. SECAM (Sequential Color and Memory) was used in eastern Europe, China and France. Like PAL it used a frame rate of $25 \mathrm{fps}$ but it processed colour information in a different way, incompatible with PAL. A PAL video played back on an NTSC television will be jerky or slow as a result of using the wrong standard. These standards were developed based on electrical power systems, which differ between regions.

These are all important considerations when working with digitized moving image material. For digital video, frame rates are much more varied, sometimes even within formats. Digital cameras from the past 20 years commonly offer the user the possibility of choosing a frame rate across several standard options.

\subsubsection{Aspect ratios}

Aspect ratios determine the width and height of an image and how it should be displayed. The aspect ratio dimensions are commonly expressed with two numbers separated by a colon ( $x: y)$, but can be displayed in two different styles. In the first style, 1 is the height of the image and the first number is the width in relation to the height, with 1 as the base. For example, an aspect ratio of 2:1 means that the image is twice as wide as it is high. But it is also common to see aspect ratios in an easier-to-read format such as 4:3. The aspect ratios of $4: 3$ and 1.33:1 are the same, just represented differently, depending on whether one wants to represent the aspect ratio with the easier-tounderstand fraction, or consistently use 1 as the base height. They are used interchangeably, but some numbers break down cleanly and others don't have an easy fraction. For example, 4:3 and 16:9 are easier to remember and type than the way their ratio breaks down in relation to 1 (1.33:1 and 1.77:1, respectively, with the trailing decimal of the first number continuing into infinity). A widescreen ratio like $2.35: 1$ is only going to break down to $47: 20$, which is not more pleasant to say than 2.35:1. It is typical for the easier-to-read fraction to be used except when comparing aspect ratios, and then it becomes easier to understand which aspect ratios are wider than others when they all start from the same base height number.

Here are some of the most common aspect ratios:

- 4:3 (1.33:1): used in traditional Standard Definition television

- 16:9 (1.77:1): used in High Definition television (and some European Standard Definition television)

- 21:9 (2.35:1): used in modern cinema

- 19:10 (1.9:1): used in IMAX films

Due to social media and mobile technology, the square (1:1) and portrait $(9: 16$, the reverse of widescreen) formats are becoming more popular as original aspect ratios coming straight from a camera or lightly edited before the creation of a final format or stream.

\subsubsection{Interlacing}

Interlacing is for optimizing perceived motion in lossy video material. It can be more commonly identified as 'annoying squiggly lines'. Interlacing issues can most easily be seen during movement between frames, where uneven lines appear in places of motion. The concept of interlacing involves each frame containing $50 \%$ of the line information required for a full picture, and having even and odd frames replay half of the information quickly enough results in a full-looking image. Interlacing 
was done intentionally at times when bandwidth was an issue, and video signals needed to be sent faster than the transfer of each full frame would allow. In contemporary video, this practice is no longer necessary and optimization for bandwidth is performed in different ways using compression techniques (see 3.2.2 Encodings for more information on that topic). Interlacing is a legacy problem, particularly when working with digitized analogue video materials.

When interlacing is performed, every other scan line of a video frame is skipped. Which scan line gets skipped depends on whether the Scan Order is set to Top Field First (TFF) or Bottom Field First (BFF).

\subsubsection{Pixels}

Video is made up of a series of image frames (and sometimes audio frames). Each image frame is made up of pixels. The height and width of video is usually described as the pixel height and pixel width. While pixels seem like a simple concept, there is a lot more going on behind the scenes than one might assume. This section will cover a few examples of this: chroma subsampling, pixel bit depth, and pixel aspect ratios.

\subsubsection{Chroma subsampling}

Chroma subsampling is the process of encoding frames of video that save less information about chroma (colour properties) than luma (perceived brightness) in each pixel.

Here are some common chroma subsampling patterns used in preservation:

- 4:4:4 This is not actually subsampling at all, but equal capture of luma and chroma fields.

- 4:2:2 This is subsampled at half the full potential chroma across two fields and full luma. This is broadcast standard.

- 4:2:0 This is subsampled at full luma and half of one chroma field. This is also common in production environments and saves space for transmission.

Remember that in $\mathrm{YCbCr}$ video, chroma is in both the $\mathrm{Cb}$ and $\mathrm{Cr}$ channels so each gets subsampled.

\subsubsection{Pixel Aspect Ratio}

Something important to understand about video is that pixels can be represented using different aspect ratios. This is not the aspect ratio of the overall picture, but the size of the individual pixels themselves that make up each video frame. There are a few concepts to understand when working with this issue.

- Pixel Aspect Ratio: The horizontal to vertical ratio of each, rectangular, physical pixel.

- Storage Aspect Ratio: The horizontal to vertical ratio of solely the number of pixels in each direction.

- Display Aspect Ratio: The combination (which occurs by multiplication) of both the pixel aspect ratio and storage aspect ratio, giving the aspect ratio as experienced by the viewer.

For the most part, this is not an issue that requires deep comprehension when working with audiovisual material. However, if during the quality control process, videos are appearing to be 'squished' and either too thin or too wide, it may be worth investigating what the intended frame aspect ratio and pixel aspect ratio are, and whether there is a discrepancy between the container and the codec with the material. Playing the material back in different video players may produce different results. 
Some layer of media archaeology will have to happen here to understand what happened with these materials: were they digitized to an incorrect specification, or was the material changed to a different media container? These are actions taken on the file that could shift how they appear and replay using contemporary media players (Rice, 2015).

\subsubsection{Timecodes}

Timecodes are a tricky subject but crucial to archival video, especially when working with broadcast media. The purpose of timecodes is simple enough: timecodes assign a number to each frame, following the format of hours, minutes, seconds, and frames (HH:MM:SS:FF). These hold a lot of significance, especially in video production environments.

Timecodes can be 'burnt' into the frames themselves, meaning they are irremovable and appear on screen for each frame. Otherwise, timecode data is stored within the file, either as part of the frame or attached as a separate track. If timecodes are stored as separate tracks, it is important to make sure this data is saved when doing digitization or transcoding work, because it can easily be skipped or removed.

Here are the most common timecode formats to be aware of when working with preservation material:

- BITC: Burnt-In Time Code, data that is stored as part of the image frame. This cannot be removed.

- $\quad$ LTC: Linear Timecode, data stored as a separate audio track.

- VITC: Vertical Interval Time Code, data is stored in the 'vertical blanking interval' of a video track. This means the data is stored as part of the video stream, on a single non-visible scan line. 


\section{Practical Best Practices}

General digital preservation practitioners should have a practical grasp of how to responsibly handle audiovisual materials. This section focuses on different concepts of digital preservation and how best to handle audiovisual materials. It does not seek to comprehensively cover digital preservation basics; rather, it introduces important concepts to consider when taking on an audiovisual preservation initiative, and provides a foundation explaining the appropriate steps to take depending on the organization's priorities and constraints.

It covers the following categories, structured from the largest and most conceptual to the smallest and most technical:

- Collaboration

- Ecosystems and workflows

- Storage

- Formats

- Metadata

- Transcription

- Capturing material

- File validation

- File characterization

\subsection{Collaboration}

Digital preservation does not exist in a vacuum, and audiovisual preservation is no exception to this. Many organizations and technical interest groups establish best practices and standards providing a framework within which preservationists perform their work.

Collaboration can come in many shapes and sizes. Some collaborations come about through serving on working groups of organizations dedicated to audiovisual preservation, others come from working together across departments at a medium-sized organization. Collaboration can come in many shapes and sizes. It is important to remember that collaboration it is not limited to attending annual conferences and producing reports: collaboration can be discussing workflows with similar institutions, contributing to open-source software through writing code or providing testing feedback, consortial-based funding models, having skill-sharing or lunch-and-learn groups within your organization to share new skills or learning, or contributing to metadata standards and guidelines.

Using and working with open-source software, whether users are aware of it (through obviously open-source software applications) or not (through proprietary software powered by open-source software), is an essential component to working with audiovisual files. Open-source software is not just free to use, but free to participate in. This can be by adding to the software, remixing it, testing it, making it part of a larger software. From an individual creating a GIF for sharing online, to the biggest companies in the world, people rely on the cumulative, collaborative effort of open-source software to create, migrate, modify, and correct digital audio and video files. Archival institutions are no exception to this. The largest and most widely used example of this is the FFmpeg suite of tools used to handle and process audiovisual files. 
Contributing to the commons is a method of furthering research and supporting collaborative efforts large and small. Collaboration can seem daunting when you feel you don't have the expertise to contribute to the wider discussion, but all contributions to open learning and working can result in another person being helped to gain a greater understanding of workflows and processes.

\subsection{Ecosystems and workflows}

Audiovisual materials have to reside in the context of other archived materials, even for institutions that seek to primarily collect audiovisual content. These materials may not fit conveniently into existing ecosystems and workflows, and care and analysis will be required when attempting accessioning, curation, cataloguing, and other common archival tasks. Specific workflows may have to be designed to adequately handle audiovisual material separately from traditional textual or photography-based material.

As much as possible, audiovisual materials should be co-located with all other archival materials and not deemed 'special' in terms of preservation storage or access to materials. This may require shaping your preservation ecosystem to fit these materials, or it may mean making compromises with the audiovisual materials in terms of storage, access, depth of metadata, or other recommended practices outlined in this report. Having an inclusive and cohesive workflow for material will help it continue to receive attention and support over the long-term lifecycle of the digital objects, not leaving them siloed without adequate care. This is especially important to think about when generating and allocating financial resources. A large grant-awarded project to create a repository exclusively for video materials is not as useful in the long term as a method of getting existing repository infrastructure up-to-date and able to adequately handle audiovisual files. This will better support emerging collecting areas such as massive research datasets, email, and web archives, in addition to the benefit of keeping all materials together.

The Issues section of this report covers some of the complications that can arise when fitting audiovisual materials into existing ecosystems and workflows, such as storage, time, assessment requirements, and hardware.

\subsection{Storage}

Storage is a significant component of any digital preservation system, but audiovisual content tends to raise storage requirements to an exponential level. Solutions that may work for small-to-medium archives with primarily textual or photographic documents are not sufficient for audio or video assets. Approaching audiovisual content is usually the point at which archivists are required to think beyond spinning disk for their storage solutions. Audiovisual assets can overwhelm existing collections.

Budgeting for storage costs should be one of the first considerations when taking on a preservation project involving audiovisual materials. When it comes to planning and budgeting for storage, involving institutional IT experts early and often can help alleviate issues in the future. This should be a collaborative effort.

When thinking of scale and planning for capacity, it helps to consider that each video file will contain somewhere between 24-60 frames per second and each frame is the size of a single image. For a digitized PAL VHS tape that runs for an hour, this is the equivalent of 90,000 images.

Below are some commonly used storage options and recommendations for what to consider when working with each of them. Overall, a combination of methods should be used to keep preserved content secure, replicated, and accessible for the long-term future. 


\subsubsection{Spinning disk}

For small archival collections, digital objects can fully live on spinning disk (otherwise known as Hard Disk Drives or HDD), following other best practices associated with storing files, such as multiple copies in multiple locations, migration assessment and planning, and institutional security practices. Special care should be taken to ensure that the files stored on spinning disk frequently pass fixity tests.

\subsubsection{Tape}

Another storage option that is popular with audiovisual material collections is using tape. Linear Tape-Open (LTO) is a preservation storage option that is particularly well-suited to large-sized preservation assets like audiovisual files. LTO is a more cost-effective and reliable option for storing a lot of data on a small footprint, both in terms of physical space and environmental impact. The data tapes are able to be 'at rest' and not rely on constant electrical usage, like other options. Tapes at rest should still be kept following basic archival storage practices for physical materials, and require consistent temperature and low humidity. As technology has advanced, new generations of LTO tapes have been launched, providing ever greater volumes of storage. Backwards compatibility of LTO drives is limited to one or two generations. As with other storage media, LTO tapes will need to be refreshed to current generations.

\subsubsection{Cloud}

Preservation storage is increasingly moving towards cloud-based solutions as these become more accepted, cheaper, and easier to manage. However, a major concern associated with cloud-based storage as it relates to audiovisual materials is an institution's ability to process and migrate very large files into the cloud. It is also important to consider bandwidth speed mixed with time and any costs associated with pulling content down from cloud-based storage providers.

Cloud-based providers are using one of the two examples above, or a combination of these technologies.

\subsection{Formats}

Formats are possibly the biggest consideration when it comes to audiovisual materials. There is no, and cannot be, just one standard that is accepted industry-wide as the best moving image or sound format. As discussed in $\mathbf{3 . 2}$ Audiovisual file structures, audiovisual files are not made up of a single format, but multiple formats working in harmony together as a container and one or more encoded streams of data.

Instead of seeking out which format is unequivocally the best, it is important for preservationists to consider what makes the most sense for their institution and for the content set to be preserved and accessed.

When discussing formats, it is important to distinguish between digitized audiovisual material and born-digital audiovisual material. For the former, a preservationist has a massive role in representing what the material will become when entering the digital part of its lifecycle. It is crucial that formats are chosen carefully, and that video is well-understood. If this knowledge is not available in-house, it is important to pick a video digitization vendor who is knowledgeable in preservation-grade transfers and is able and willing to explain the decision-making process behind chosen formats.

For more information on format recommendations, see Section 6 Standards and Technologies, which covers some common preservation formats, and looks at what causes preservationists to favour one over another. 


\subsubsection{Recommendation guidelines}

There are some general guidelines to consider when thinking about preservation and access formats, and deciding what is best for an institution, given constraints around capacity, time, budget, staff, and long-term goals.

\subsubsection{Open or closed}

File formats and encodings can have open or closed standards. This means that the rules around creating files, or creating software that works with files, are either available to everyone, available only through private organizations with expensive associated fees, or closed to anyone outside of a specific organization. Choosing an open format helps ensure that the ability to read and write files will be sustainable into the future.

When working with born-digital material, and emerging technologies like Virtual Reality or Augmented Reality, choosing an open format is not always an option. In that case, focus on other aspects of the format, generate documentation on practices and workflows, and collect knowledge around what it will take to recreate or emulate the media in the future.

\subsubsection{Well supported}

It helps to choose a format that is well supported on the machines you have, and will continue to have, access to and work with. This includes hardware and software used to replay formats. Many 'best practices'-level preservation formats can be difficult to handle using standard electronic equipment. If your organization is particularly under-resourced or lacks technical knowledge, it should be worth considering whether high-quality but imperfect preservation copies of video files are sufficient because of the payoff in access terms.

\subsubsection{In-house knowledge}

It is important to consider the in-house capacity of staff when deciding what formats to work with. Some moving image formats, such as DPX files, can be difficult to replay on standard computers. DPX files are a series of images, one per frame, representing motion pictures. These would have to be transcoded into copies suitable for presentation and for access, so time, resources, and staff expertise will be necessary to make that happen.

\subsubsection{Migration}

Overall, migration or 'normalization' is not recommended for audiovisual materials. Of course, there are some exceptions to this. Born-digital materials should stay in the format they were created in. Attempts to take born-digital material and convert it to a preservation format may result in a loss of quality, significant increase in file size, or both. Because audiovisual files have so many properties beyond those which is seen or heard, it can be a dangerous process to migrate them.

It is also important to keep in mind that especially with born-digital files, there may be aspects that give insight into the provenance or artistic intent of the files, and migration risks losing that information. For example, files may have retained embedded metadata about the type of software and version that was made to produce it. Especially in the context of art conservation or digital records processing, extra care needs to be taken that these tiny details - sometimes only available by looking at the file in a hex editor - are retained in the preservation process, because they could be significant in the future when replaying material, especially if the material is meant to be replayed within a specific context, such as an author's laptop or in a museum setting.

Digital-to-digital migration may be attempted when there is an understanding and assurance that the content and significant elements of files will not be lost. 
An example of ensured migration is the MediaArea project RAWcooked ${ }^{2}$. This open-source tool takes DPX files and converts them into an FFV1-encoded data stream wrapped in a Matroska file. This supports storage, as the (lossless) compression algorithm and self-describing metadata components in FFV1 and Matroska (MKV) make it a better preservation storage candidate. But the process is reversible, and the FFV1-encoded Matroska file can be converted back into DPX files and the checksum will be identical. In an example like this, it is safe to perform a migration and the preservationist can feel confident that their work did not erase any significant file properties.

\subsection{Metadata}

Metadata is a crucial aspect of preservation. For audiovisual materials, there are metadata standards that fulfil unique aspects of the formats. In general, institutions should rely on whatever standards they currently use within their ecosystem, as outlined in 4.2 Ecosystems and workflows.

\subsubsection{Standards}

Audio and moving image materials rely on the dimension of time to be experienced, which adds an extra dimension to the associated descriptive metadata. Several standards exist specifically to describe the content of audiovisual materials.

- AES57: This Audio Engineering Society standard is for audio object structure metadata.

- EBUCore: An extension of DublinCore developed and maintained by the Technology \& Innovation team at European Broadcasting Union.

- EN 15907: This European Standard specifies a set of metadata for the description of cinematographic works, as well as a terminology for use by parties wishing to exchange such descriptive metadata.

- PBCore: An extension of DublinCore developed by United States public broadcasting communities and maintained by the WGBH Educational Foundation.

- VRA Core: A descriptive metadata standard developed and maintained by the Library of Congress Network Development and MARC Standards Office in partnership with the Visual Resources Association External Link. This metadata standard is focused on general visual artworks.

\subsubsection{Embedded versus sidecar metadata}

Preservationists must decide how to store metadata associated with audiovisual content, and this is contingent on the capabilities and limitations of chosen file formats. With audiovisual materials, some content can be embedded within the files themselves. For others, associated metadata must be placed in a 'sidecar' metadata file that sits alongside the preservation assets or in a preservation system database.

\subsection{Transcriptions}

Some audiovisual materials come with closed captioning or displayed text, most commonly seen on live television broadcasts. This material is captured in the Line 21 section of the video format and must be activated by a viewer using an assisted device or by adjustment of the default broadcast settings.

${ }^{2}$ RAWcooked https://web.archive.org/web/20200816002246/https://mediaarea.net/RAWcooked 
Other video material will have subtitle information that plays alongside the video, either embedded into the video file or coming from sidecar files formatted to store subtitle text and timecodes.

If audiovisual material does not come with transcriptions readily available, they will have to be prepared. While images with written text can have the meaning extracted into a text file, this is not reliably available (at time of writing) for audio materials at an appropriate quality level. Transcription by computer is not yet sufficient, as while it can give a gross approximation of the content, automated transcription is not prepared to handle accents or niche topics in a way that would fulfil the accessibility guidelines for people with hearing difficulties. For moving images, there may be a need to contain transcriptions not just of the soundtrack, but also of an explanation for what is happening on-screen during the runtime. This can vary wildly, and largely depends on the material, organization, access, rights, and country jurisdiction.

\subsection{Capturing material}

Audiovisual material may, at times, need to be converted from one format to another. Content may have to be migrated from analogue to digital, or from digital file to different digital file. This is a significant preservation event because it shifts everything that makes up the content. In a preservation context, it can be considered a PREMIS event that changes the digital provenance. In the context of museum conservation, it can be considered a significant conservation treatment.

\subsubsection{Analogue to digital}

Getting assets off their analogue carrier is an important and crucial step for the preservation and access of most analogue material. Experts speak of this in the terms of a crisis, we are in a 'magnetic media crisis' (Casey, 2015). We are truly at end-of-life for all magnetic media carriers. There is a limited amount of playback devices and the analogue material life cycle timeline is coming to a close due to physical entropy naturally caused by the media. The media itself that the valuable archival content lives on is slowly decaying, and the estimated length of time that most magnetic material is estimated to function for is in the 30-50 years range. For all magnetic-based analogue or hybrid material, the end of the life cycle is now. Because of this, conversion to a digital medium is urgently necessary.

\subsubsection{Digital to digital}

Material that has been digitized, or was digital from inception, may, at times, require intervention and migration from its current format to a format that is more sustainable for the future. This can depend on different situational factors. If digitized materials were digitized at the highest quality as a lossless format, a decision may be made by an institution to apply a lossless compression to the audio and video streams in order to create an overall smaller file size footprint. Another case for transcoding uncompressed files to a losslessly compressed encoding is that the encoding may provide additional preservation practices embedded within the encoding, such as checksums available at a more granular level to help verify content in the future or pinpoint where data has been lost more specifically than at the file-level.

\subsubsection{Streaming}

Ideally, audiovisual content that is available as a stream or embedded into software or a webpage could be preserved through an original file-based source. In that case, the material would be handled as documented throughout this report and its predecessor. 
If the content is available on the web, a web archiving tool could be used to capture the audio or moving image material. Examples of software for this purpose include the Webrecorder ${ }^{3}$ capture application or Wayback ${ }^{4}$, the open-source web archiving engine that runs the Internet Archive's Wayback Machine. Another option would be to download the website in full using the command line tool 'wget' in an effort to retrieve embedded files. This is the preferred method if the importance of the audiovisual material is significant within the context of its surroundings.

However, the ability to retrieve files may not always be available, especially in the case of community-based or activist-based archives. In this case, preservationists should seek to capture the full screen while streaming the media at the best quality possible.

An issue with successful screen capturing is that some video content will be protected by Digital Rights Management (DRM) software or other anti-piracy efforts. In this case, material attempted to be captured will merely show up as a blank screen after the recording is saved. There is not a reasonable way around this.

A concern as large as the technical challenges of stream capturing is the ownership and management of copyright of the captured material, which is covered in $\mathbf{5 . 1}$ Legal and ethical considerations.

\subsubsection{Working with vendors}

In many situations, organizations do not do their own format migration themselves, but rely on vendor services that have the sufficient technical knowledge and hardware to create preservationgrade digital surrogates for analogue materials. When this is the case, preservationists should research their ideal target formats, discuss them with the digitization vendor, and focus on performing quality control checks on files upon their return. If possible, automated quality control by checking files for their most crucial technical characteristics should be performed. In addition, visual 'spot checking' of files should be carried out with at least $15 \%$ of the returned files getting some sort of visual inspection within the window of time when complaints can be formally lodged (typically 30-60 days).

\subsection{File validation}

Digital files are complicated. Using tools that reinforce confidence that files are truly what they say they are is important to the preservation of audiovisual files. As mentioned in previous sections, it is essential that audiovisual materials end up in a digital form, either for preservation or for access. Preservationists have control over the file format and should take care to ensure that the files are valid according to their specification and any other institution-based policies the files should adhere to.

An example of conformance to the file format's specification is verifying that all of the bits of data that make up a file format are appropriately arranged; fundamentally, it is ensuring that the files are not broken in any way.

Unfortunately there are limited options for the validation of video formats. For Matroska (MKV), LPCM, or FFV1 encoded files, the open-source tool MediaConch ${ }^{5}$ can be used to validate files against their specification at the byte-level. MediaConch can also validate files against an institution's

\footnotetext{
${ }^{3}$ Webrecorder https://web.archive.org/web/20200830194722/https://webrecorder.net/ ${ }^{4}$ Wayback https://web.archive.org/web/20200811061624/https://github.com/internetarchive/wayback/ ${ }^{5}$ MediaConch https://web.archive.org/web/20200810055951/https://mediaarea.net/MediaConch
} 
particular policy. An example of conformance to an institution's policy on a particular file format would be if a set of digitized material was intended to all be of a specific pixel dimension. If all the files in a set conform to $640 \times 480$, but one file has the dimensions $640 \times 380$, then that file is not adhering to the rules set in place by an institution and the expected quality of an archival asset.

Because audiovisual files are packages with multiple elements, it is important to investigate files and understand how their significant technical characteristics are asserted, and which elements are making those assertions.

Files should also be considered against their limitations. Here are a few questions to consider when determining a file's suitability for its intended purpose: preservation or for access.

- Can metadata be embedded into the file?

- Can this file hold subtitle or captioning data?

- Can this file be played back on devices?

- Does this file play well on the web?

\subsection{File characterization}

Understanding the most valuable technical characteristics of audiovisual materials can be done through characterization, and the extraction of technical properties. Because audiovisual files can be so complicated and have so many components, it is important to understand the ways in which they can go wrong. Many of the essential characteristics of an audiovisual file are outlined in $\mathbf{3 . 2}$

\section{Audiovisual file structures.}

If an audiovisual file is not looking or sounding the right way, the best thing to do is to look at the its technical characteristics. Extracting this information at the beginning of the preservation process can support the quality control process and help determine if the file has changed or been malformed in a specific, unexpected way.

Information about files can be extracted using tools dedicated to this purpose, such as Medialnfo, FFmpeg, or Exiftool (for image-based materials). Medialnfo and Exiftool are available as graphical software applications (GUIs), and FFmpeg is available only on the command line.

\section{Issues}

Audiovisual materials can cause practitioners to question their existing practices and workflows, and there are specific 'gotchas' that make this material more challenging. This section brings those issues to light and increases awareness of some of these complications, so that preservationists can be adequately prepared to handle them. It summarizes some of the primary issues and concerns associated with audiovisual materials, and how they are distinct from other materials within the context of digital preservation. Covering some of the most pressing issues specific to audiovisual preservation, this section will look at legal and ethical issues, geospatially aware media, assessment requirements, hardware, financial issues (including storage and time) and the tension between preservation and access.

\subsection{Legal and ethical considerations}

Audiovisual materials conform to the same rights and regulations as other archival materials depending on local jurisdiction, but can differ in some significant ways that are worth drawing attention to. 


\subsubsection{Significant migration}

Various professional organizations associated with the preservation of moving image and sound materials have ethical codes surrounding this work, particularly around migrating material and thus significantly changing its essence. These have been summarized in Digital Archiving of Film and Video (Jarczyk, Kromer, and Pfluger, 2020). In short, preservationists should seek to protect the integrity of archival materials, restore and preserve without alternating original materials, and treat materials with respect.

\subsubsection{Environmental concerns}

Due to the size of digital audiovisual materials, their environmental footprint can be significantly more substantial than that of other archival digital objects. When it comes to the preservation of large sets of audiovisual assets, prioritizing workflows that support less energy usage is important. See 4.3 Storage for understanding these impacts and how to identify which storage mediums are best suited for large collections (as a bonus they are usually less expensive too). Organizations such as Project $\mathrm{ARCC}^{6}$ can provide additional information and resources on this subject.

\subsubsection{Capturing the ephemeral}

Moving images provide a primary way to capture dance choreography, and are the closest possible connection to an inherently ephemeral medium outside of a live performance or recreation of exact movements. Because of this, there is a close connection between audiovisual history and dance history. Worth emphasizing is the copyright status of dance movements and how this is different from how copyright works for other, more physical, materials.

For additional information on working with dance materials, Dance/USA has a section on copyright in their Artist's Legacy Toolkit (Dance/USA, 2020).

\subsubsection{Streaming or embedded content}

Digital video is evolving to include ephemeral media streamed through social media. Sometimes this video can be retrieved and downloaded through the application doing the streaming. This may be available to the general public, but is sometimes only available to the producer of the media (and only if the producer of the media is able to access their account).

When seeking to preserve material that has been streamed over the Internet or embedded into web pages, it is important not to forget that, like all archival material, this should be collected with ethical considerations. Organizations such as Documenting the Now ${ }^{7}$ and WITNESS ${ }^{8}$ can provide additional information and context through an ethical lens. Documenting the Now works towards creating tools that better capture social media content while considering ethical practices and WITNESS is an organization focused on helping people use video to protect and defend human rights.

\subsection{Geospatially aware media}

Digital video is much more complex than most current resources attempt to cover. The fundamentals of digital video covered in Section 4.2: Audiovisual file structures summarize the typical technical characteristics of audiovisual files. Virtual reality, augmented video and (sometimes) $360^{\circ}$ video have an additional dimension to consider: physical space. How is this media meant to be situated in physical space? This is typically up to the media carrier to embed within the files. Extra care should be taken and research conducted to ensure that this information is not lost

\footnotetext{
${ }^{6}$ Project Arcc https://web.archive.org/web/20200414203221/https://projectarcc.org/

${ }^{7}$ Documenting the Now: https://web.archive.org/web/20200819122339/https://www.docnow.io/

${ }^{8}$ WITNESS: https://web.archive.org/web/20200903143046/https://www.witness.org/
} 
when preserving the files, and it is especially important not to migrate these formats to another medium. As outlined earlier in the report, born digital audiovisual materials are best left in their original format rather than migrated to a format recommended for general use.

\subsection{Assessment requirements}

A requirement unique to audiovisual material, particularly any material that is analogue or living in an analogue carrier, is that migration to an accessible format must happen before any collection assessment. Digitization has to happen before processing or appraisal, curation or selection, assessment, even acquisition, can be done. Oftentimes, processing archivists are entirely limited to what is physically written on the carrier or any attached physical media containing relevant descriptive metadata. This will require preservationists to rethink workflows in significant ways, and many tasks that usually happen later in the process must happen up-front, before any processing begins. Even the minimal processing that is required just to get the material in an accessible format is very costly and time-consuming. Preservationists should focus on assessment and coming up with a business plan for working with material, and do as much research as possible on the contents of analogue files, in order to make the best choices for preservation.

\subsection{Hardware}

Access to analogue or hybrid analogue-digital materials is bottlenecked by functional hardware. This old hardware has a lifespan limited to how many transfers it can perform before wearing out to the point of no repair. Even if machines can be repaired, there is both a growing lack of expertise available to fix the equipment, and an increase in associated costs (Toth, 2019). Expertise associated with working with old hardware must be available on your staff, through a steady consultant relationship, or through a reliable vendor relationship. Costs for this relationship are increasing as the expertise and available hardware become increasingly rare with age.

Legacy hardware must also learn to be compatible with modern hardware and associated software. There must be consideration for what is out of date on modern machines running modern operating systems, and the gap bridged between what the old machines and new machines can do together. What is the in-between for getting these technology components to work together? This problem is not limited to the hardware but affects software components as well, as some formats may rely on software that was previously commonplace but now is no longer available. And, if the software is available, it may not be able to run on modern equipment.

\subsection{Access versus preservation}

Access is not a problem particularly unique to audiovisual materials, but there is a difference between preservation and access formats, and they must be handled differently. Preservation copies of files can be a hindrance to work with. They are very large, and some recommended preservation formats cannot be replayed on a personal computer. Some archives work with this concept of a middle-tiered file, known as a 'mezzanine' file, that is higher quality than a normal access file but lower than the preservation copy. Coming up with the right solution for an institution can be challenging.

\subsection{Financial considerations}

Working with audiovisual materials has a bigger impact on preservation budgets due to many of the Issues mentioned above, such as increased storage, legacy hardware, modern processing hardware and time.

As highlighted in $\mathbf{4 . 3}$ Storage, storing audiovisual materials requires more thought and planning than storing other materials due to the file size being so much larger than images or text-based 
documents. This means that archives that have been collecting and preserving material for a long time but are just getting started with sound or moving image content may have to rethink their entire approach to storing digital objects.

Unlike some other archival materials, audiovisual materials have the additional dimension of time. The media cannot be experienced without a predetermined, set time as a component. Time is not an issue just for the consumption or review of the assets, but the preservation work also takes additional time due to file size. Processing materials, sending things through predetermined workflows, generating checksums, and gathering metadata can take a lot more time than with other formats. Capacity planning must include dedicated staff time for this purpose. 


\section{Standards and Technologies}

Because of the complexity of audiovisual file formats, understanding and making an informed decision around standards and technologies is one of the most confusing and difficult decisions that a preservationist has to make. This is compounded by the need for digitization of fragile, endangered analogue material and the significant preservation action required to change analogue material into digital format. This section covers current standards and technologies based on existing research and recommendations, and explains which are most suitable for each type of media and type of institution. It will cover the pros and cons of some common preservation formats, and what causes preservationists to favour one over another.

\subsection{Audio}

There is a general consensus in audio preservation communities, as to how sounds should be preserved, which is in an uncompressed format, wrapped into a container ${ }^{9}$. This section will cover the primary method, WAV (including Broadcast Wave Format), and another popular method, FLAC.

The general recommendation for uncompressed audio is that it is sampled at $96 \mathrm{kHz}$ with 24-bit audio sampling, although high-quality music recordings are often digitized at $192 \mathrm{kHz}$. The lower threshold for a high-quality audio sampling rate is $48 \mathrm{kHz}$. Exceptions to this are when the original material sampling rate is lower than this, and there is no benefit to increasing the sample rate when the fidelity is already low.

The dominant container format is WAV. Sometimes FLAC is used as a lossless encoding for its costsaving benefits. See below for more information about each of these formats.

\subsubsection{Uncompressed Audio and WAV}

Uncompressed audio streams typically use Pulse Code Modulation or Linear Pulse Code Modulation to digitally represent sampled analogue audio signals ${ }^{10}$.

Uncompressed audio streams are generally placed into a WAV file format. This is a small wrapping container that stores the audio and little other information.

For preservation purposes, this format is sometimes extended as Broadcast Wave Format, or BWF. BWF allows for additional metadata to be embedded into the file, allowing the file to be selfdescribing. These WAVE 'chunks' support adding metadata, including information about the original broadcast extension, quality, the MPEG audio extension, the peak envelope, additional XML, and other data.

A downside to WAV files is that they have a size limit, which is $4 \mathrm{~GB}$ of audio data per data chunk. Another extension to the WAV format, RF64, allows file sizes to be exceeded and is a popular solution to managing preservation audio.

\subsubsection{FLAC}

FLAC is an open-source audio encoding format that supports lossless compression. It represents both the container format for holding the file together and the encoding format for the audio

\footnotetext{
${ }^{9}$ See IASA 2009 Guidelines on the Production and Preservation of Digital Audio Objects (web edition) https://web.archive.org/web/20201001081959/https://www.iasa-web.org/tc04/audio-preservation last accessed 23/09/2020
}

${ }^{10}$ See https://en.wikipedia.org/wiki/Pulse-code_modulation 
stream. As a format, it is well supported across all modern operating systems, including mobile operating systems. In a preservation context, FLAC tends to take a backseat to the de-facto standard of storing LPCM audio inside WAV files.

FLAC produces an audio file that is lossless compared to the uncompressed audio, but can reduce the file size by $50-70 \%$. This can have a large impact in overall storage costs long term when compared with WAV files. FLAC files do not have a size limit and can be larger than 4GB. Additional preservation benefits include that FLAC also supports metadata tagging, embedded images (e.g. for an album's cover art), and CRC-32 checksums per frame for additional integrity-checking capabilities.

\subsection{Video}

Unlike for audio, there is no well-established standard that is generally agreed-upon by experts. This can make decision-making around video standards difficult. This section features some of the most common video formats, listed alphabetically, and articulates what makes them popular and looks at the situations in which they are most likely to be recommended. They are referred to by their most prominent descriptive factor, often the container.

\subsubsection{DPX}

Digital Picture Exchange (DPX) is a file format developed by SMPTE, a professional organization and standards body. It is typically used as a target format for digitizing film and is common around the world in digital intermediate post-production facilities and film labs. A DPX file represents a single frame, so a sequence of files are necessary to represent a digitized film. DPX sequences can be saved in both the ungraded (a raw capture with no colour editing) and graded (a preservation master with final colour edit) versions.

\subsubsection{MKV}

The Matroska container and FFV1 video codec are in the process of being standardized by the Internet Engineering Task Force (IETF) for use as a preservation format. This is the result of research and initial funding from the European Commission's PREFORMA Project, which focused on ideal target preservation file formats (PREFORMA, 2018). Matroska is a container that stores CRC32 checksums for every portion of the container, so integrity of the video and audio streams can be checked even if the embedded metadata changes, instead of the checksum invalidating for the entire file. The target video encoding, FFV1, also includes more granular checksum features than in most other media carriers, and can store checksums at the frame or even frame slice level so that errors or bit rot can be precisely identified.

\subsubsection{MXF}

Material Exchange Format (MXF) is a video container format also established by SMPTE. In a preservation context, it has largely been championed by the United States Library of Congress and used for large-scale, mostly automated, video digitization. For preservation purposes, JPEG2000 is often intentionally used as the video encoding within the MXF container, although the MXF container can wrap several other video encodings such as the native editing format, Avid DNXHD. Video streams, audio streams and timecodes are sometimes split between separate MXF files. MXF has also been used as the audio and video packaging format for Digital Cinema Packages (DCP), the format used for playback of movies in cinema theatres. DCP is a more formal folder structure that contains MXF files. Professional video cameras can output MXF files as their born-digital camera originals and archives have encountered these files.

For more details on using MXF in a preservation context, see Sustainability of Digital Formats: Planning for Library of Congress Collections: Material Exchange Formats (Library of Congress, 2020). 


\subsubsection{MOV and AVI}

Perhaps the most common standard for video preservation has been not using any specific codec at all, and storing the video stream as an uncompressed data stream wrapped inside an Apple Quicktime container file (.mov) or a Microsoft Audio Video Interleave container file (.avi). The typical video content inside is either an 8-bit or 10-bit pixel depth uncompressed stream of data with a 4:2:2 chroma subsampling (see 3.2 Audiovisual file structures for an explanation of those concepts). For the digitization of lower-resolution media such as VHS tapes or Standard Definition broadcast, 8bit level pixel depth is perfectly acceptable.

Quicktime container files can also often contain data encoded with the Apple ProRes codec, as it is a popular export option for video editing programs on macOS.

\section{Case Studies}

The preceding sections of this report provide guidance on best practices and issues associated with the preservation of moving image and sound materials. This Case Studies section illustrates a range of scenarios in which preservationists find themselves working with audiovisual material, from large institutions to small community-driven archival projects. They will describe how specific organizations have implemented the preservation of audiovisual objects in the context of their environment, resources, and mission.

\subsection{International Atomic Energy Agency}

The International Atomic Energy Agency (IAEA) is an international organization that promotes the peaceful use of nuclear energy and inhibits its use for military purposes. The IAEA was established in 1957 as an independent organization within the UN family, and reports to the United Nations General Assembly and Security Council. Its headquarters are in Vienna, Austria, and it has remote sites in seven additional locations across the world. As an international agency seeking to educate the world about nuclear energy, the IAEA also has a large collection of films produced on this subject held in multiple locations by multiple departments, mostly in analogue formats.

The content on this media showcases the IAEA's past work in promoting peaceful use of technology, hosted or sponsored events, conference proceedings, speeches and interviews from early staff members, and denuclearization efforts across the world. As these materials exist in analogue formats, they are not available to be distributed through modern means on the web and they risk being lost due to decay of the original analogue formats.

The archives division at the IAEA established an internal business case for the inventory, digitization, and long-term preservation of these audiovisual materials. Internal advocacy is a crucial element to beginning audiovisual preservation projects, but can also be a labour-intensive and lengthy process, depending on the size and complexity of an organization.

The IAEA archives is able to articulate all of the reasons why preservation of these audiovisual materials is a task worth investing in. They drafted an internal report that emphasized the following points:

- External researchers, journalists, and departments will be able to better find and access audiovisual material;

- Audiovisual material will be used more broadly, helping to promote the work of the Agency; 
- Having an available inventory and catalogue will enhance advocacy work with rich digital resources;

- The digitization of audiovisual material currently on analogue and legacy digital carriers will ensure its preservation beyond the life of the carriers themselves;

- Storing audiovisual material in line with long-term preservation requirements will mitigate risk of loss of audiovisual material;

- It will reduce the time needed to handle requests for historical audiovisual material;

- Digitization will reduce the need for physical items to be stored in an easy-to-access manner;

- By storing all audiovisual metadata in a platform with a single point of access, material that is currently spread out through different Agency units/divisions will be able to be searched for efficiently through a single interface;

- Publishing of more historical visual content will help build the public visual history of IAEA;

- Standardized online public licensing, citation rules and authoritative metadata will establish a stronger visual and academic identity. It will ease control of IAEA intellectual property.

A business case advocating for audiovisual preservation should not only contain the positive aspects and benefits to preservation, but emphasize disadvantages and risks as well, so that there is a well thought-out and clear understanding of the situation, approached from all angles.

Some negative aspects presented as part of the business case include:

- The creation of a budget and risks associated with that budget;

- There is a risk that there will be a large increase in requested access to materials, which puts a strain on the staff who currently manage this work;

- Issues focused around copyright and the accuracy of that information, and management of any take-down requests or dealing with material in which the copyright is ambiguous;

- Increasing digital storage, and the resulting increase in annual costs long-term;

- Time. The project will likely take 12-24 months, possibly more;

- Incomplete, or poor quality, control measures, resulting in poorly digitized archival materials.

The IAEA archives team planned out the different stages of an audiovisual preservation initiative into three distinct categories:

- Inventory. Figuring out what holdings your organization has is a task that may seem simple, but can take a lot of time and effort. If the collections are geographically dispersed, such as is the case with the IAEA, it can be difficult to understand the collection as a whole. If the collection contains many different formats, or in-house knowledge about the nuances of different formats is lacking, it can become a longer process than intended.

- Digitization. Digitization requires an approximately one-time cost for an organization, and can either be outsourced to a digitization vendor or performed in-house, if the organization 
feels it is worth investing in the equipment and training. The IAEA proposed both of these potential solutions, weighing the pros and cons of each.

- Long-term preservation. This is a crucial aspect to any audiovisual preservation action plan, because the long-term funding and sustainability need to be taken into account. The responsibility of taking care of these materials needs to be a long-term, never-ending commitment.

Acknowledging the work that happens before, during, and after major preservation actions is a great way to plan for a future digitization initiative. With audiovisual material in particular, it is important to perform as much work as possible on the initial inventory of materials, to determine if there are any repeat or non-unique materials. This will save on digitization costs, which are significant in time and finances. There may be some materials that do not need to be preserved and can be deaccessioned through the act of not being digitized.

For IAEA, a benefit of digitizing the material is to make more office space available. This does not mean that the original media should be discarded, but that it could be stored in a more compact and affordable location, because regular retrieval and handling of the material on an individual basis will no longer be necessary. The original analogue carriers should be retained. This is especially important for film elements that will stay in good condition for a long time, and also for magnetic media carriers that are at a higher preservation risk. If these collections have to be split into categories, it would be better to digitize the magnetic media first and save the film elements for later, as film is a stable preservation medium, even if it is not good for access to content. IAEA digitized the film material first because it was readily available and could be outsourced to a vendor, while they have in-house equipment and knowledge to digitize the magnetic media themselves.

\subsection{Mark Morris Dance Group Archives}

Mark Morris Dance Group was founded in 1980 by choreographer and dancer Mark Morris. In 2001, the Mark Morris Dance Center opened in downtown Brooklyn, New York, to expand on the dance company's mission, provide space for dancers to learn and practise, and as an educational space for the local community. The Group's archives consist of theatre production materials, playbills, photographs, correspondence, and a collection of audiovisual content stored on various analogue magnetic media carriers. The audiovisual content, primarily U-matic and VHS tapes, contain recorded footage of dance performances not available anywhere else. This archive has been in storage with minimal inventory or cataloguing work, other than what has been needed in association with marketing initiatives.

Attempting to capture and preserve dance performance is a unique preservation challenge, because the content is inherently ephemeral in nature. An event or a specific dance cannot be captured wholly, but attempts can be made through recording video, saving ephemera such as posters or production bills, keeping costumes and stage props, dance notation, and through interviews with people who participated in the creation or viewing of the event.

Dance, particularly in the United States, has an additional consideration when it comes to archival access. Dance moves do not hold copyright in the same way as other forms of media, such as a book. Because of this, some choreographers are protective of their work and wary of giving broad access because they do not want particular moves or actions to be 'stolen'. This can present a challenge when giving sweeping access to dance collections, especially involving many different choreographers and dancers who feel protective of their particular work or style, and must be handled with some sensitivity. 
Through a collaboration with Dance Heritage Coalition (a formerly independent organization now part of Dance/USA, a service organization for performance artists, administrators, and organizations) and funding from the United States Andrew W. Mellon Foundation, the Mark Morris Dance Group was able to hire their first full-time archivist to focus on the digitization of photographs, playbills, and video materials in the collection with high preservation risk and value. This includes video material that features unique performances and is not available anywhere else.

During this three-year project, the Mark Morris Dance Group hired four part-time staff in addition to their full-time archives manager to organize and digitize print and audiovisual material on-site with equipment purchased for the project. Equipment included two desktop computers, an analogue-todigital convertor, a flatbed scanner, and analogue video playback equipment. Some materials were interviews and oral histories available on MiniDisc. Because of the fragility of the medium and lack of playback equipment, oral history and interview content on the MiniDisc format was sent to a digitization vendor instead of attempting to digitize it on-site.

The Mark Morris Dance Group did not set up all of their equipment on their own. As part of the grant-funded initiative, a local audiovisual preservation consultant was brought in to prepare and approve the purchased equipment, set up digitization stations and workflows, train staff on using the equipment, and support the development of a web-based cataloguing and access platform. With this help, the newly formed archives team felt confident that they were making the right preservation decisions. The tapes were digitized using vrecord ${ }^{11}$, an open-source software made by archivists to digitize and provide live quality control checking for magnetic media. The target format for these digitized materials was FFV1-encoded video and LPCM audio wrapped in a Matroska (.mkv) container. These materials were stored on a small replicated server hosted within the archives, as well as on a local machine for easy access, and regularly backed up to a cloud-based storage. The latter backups-generation service was performed as part of their usual IT routine, making it part of the normal workflow of the larger organization's IT services rather than an independent operation to be managed by the archives long term.

In addition to preservation, it was important to give access to these collections. The plan was to initially provide internal access for the rest of the company so that they could easily search and retrieve specific performances. This could be used by dancers to practise performances, the educational committee to help with teaching practices, or marketing to promote future work. For the access component of this initiative, another consultant was brought in to work with archives staff on developing an appropriate data model for understanding archival material presented by a dance company; creating connections between the unique works, specific events, and associated archival material. This consultant also worked on installing and configuring the chosen cataloguing and archival access platform (CollectiveAccess) and designing a front-end platform aligned with the Mark Morris Dance Group's brand.

In addition to digitization work, archivists on the archives team were focused on cataloguing each work by describing the content, including timestamps for moving image materials, and associating each digital object with the related conceptual work, specific event, location, and persons associated (including their roles).

Initial planning and funding are excellent first steps in establishing an archive for a small-to-medium sized organization for the first time, but archives are intended to be established permanently. As part of this project, the archives manager focused on creating an archive within the organization that

${ }^{11} \mathrm{vrecord}$ https://web.archive.org/web/20200626094818/https://github.com/amiaopensource/vrecord 
could be integrated into all aspects of the Mark Morris Dance Group's existing workflows and systems, such as marketing, development, education, and special performance initiatives. By integrating fully into the larger organization, the archives can become an essential service and receive financial commitment long term.

\subsection{Borthwick Institute for Archives at the University of York}

The Borthwick Institute for Archives is an archival repository and service-focused organization based at the University of York. Their holdings include the archives of the University of York, collections surrounding the Church of England and nonconformist records, archives for individuals and families of historical significance, the archives of several important composers, the York National Health Service archive, and the archives of local businesses and organizations.

The Institute collects a variety of analogue and digital materials, including audiovisual records. Many collecting organizations have separated the way they process archival material by analogue and digital, and this is also the case for the Institute. The audiovisual material being stored on physical carriers, primarily consisting of audio, are either born analogue formats that need to be converted into a digital format for long-term preservation or contain digital content that requires migration off analogue carriers. There is an urgency associated with this need for migration that is higher than that for text-based or image-based documents due to the sensitivity of the formats, equipment becoming rare, and expertise being lost over time. It is not uncommon for audiovisual materials to sit awkwardly between previously established analogue and digital workflows, where they begin as analogue assets but cross over to becoming primarily digital objects.

When the Borthwick Institute for Archives hired a digital archivist, she initially performed an assessment project to uncover and understand the scope of digital material on analogue carriers found in the collections. This ended up being an assessment of all of the analogue audio materials across the Institute.

The digital archivist sought to answer the following questions as part of this assessment project:

- What do we have?

- What formats are the materials?

- Which archives hold these materials?

- How much content is there?

The end result of the collection assessment project was a spreadsheet covering nearly 34,000 audio objects across many collections. With audio regularly coming into the archives, and with the expectation that this type of content will increase in the future, it was deemed crucial to come up with a preservation plan for the audio material and to initiate the digitization of audio material.

There was no dedicated audiovisual preservation specialist on staff. Instead, the digital archivist did an assessment of what kind of knowledge already existed among staff, and how that knowledge could be aggregated to fulfil the needs of this emerging project to manage audio at the Institute.

There is no audio archivist, but there are other departments that do similar work: Conservation, Digitization, Digital Archives, and the University of York Music department. Each department had important knowledge and skill sets to bring to the project. The Conservation team focused on the care of non-audiovisual material, but had a foundation in collections care and management, and could bring perspective on how to appropriately carry the essence of the material into a new 
medium. The Digitization team had the skills and expertise to digitize materials and were able to apply that knowledge to audiovisual material, which they previously had not worked with. The Digital Archives department (one person) was able to lead the initiative and bring knowledge of the care and structure of digital files, metadata, and long-term preservation planning. The Borthwick Institute for Archives is responsible for collecting materials across the University of York, and the music department was interested in working with this material and ensuring its preservation. They had the knowledge of formats, context for some of the collections, and equipment, but no experience in archiving.

In addition to equipment from the University of York's Music department, the Institute was fortunate to have audio equipment that could perform the digitization work. This equipment was limited and did not fit the wide variety of formats available in all of the collections, but it was possible to start the digitization process and save other formats to be outsourced at a later time or digitized in-house later when equipment could be purchased. The existing equipment was used for 'ad hoc and on demand' requests from researchers.

The 'on demand' digitization resulted in creating 'ad hoc' digital files that had been digitized by different people over time with different skill levels and to different standards. There was no specific standard for materials that have been digitized, because they had been digitized across many different methods, eras, and sources. In addition to the ad hoc digitization for research requests, audio had been outsourced to different people or companies, digitization performed as part of a project-based initiative, and other workflows. There were also materials that had not been sent to the archive, and workflows that were never documented.

The departments came together to create an audio digitization workstation, relying on knowledge from the University of York music department to provide guidance, and the Conservation department to provide guidelines for how to handle materials in a responsible way. Documentation is a crucial aspect to any digitization work, and a seven-page guide was drafted for digitizing audio cassettes at a very basic level, to document and provide coverage for anyone coming in to pick up the work without any previous experience.

By working collaboratively across departments, the Borthwick Institute for Archives was able to make a 'best efforts' attempt to organize what was available in-house, using in-house staff and the knowledge that they had available to them. Rather than framing this as a 'project' that would interrupt regular work duties, the Institute's staff was able to work on digitizing when they had extra time.

This project came together to accomplish the following goals: understanding the breadth and depth of the audio content across archives collections, establishing a new workflow for material coming in, setting up in-house digitization, establishing a plan for handling film and video material outside the Institute, setting up audio for successful transfer over to the digital archives, and creating skillbuilding and capacity-building opportunities across departments.

The most important lesson to be learned from the Borthwick Institute is that 'perfect' is the enemy of good. Due to the large backlog of material and the incoming stream of new audio and video material, it is important to start processing and managing these materials with the resources that are currently available. Other important takeaways are that staff can learn by doing, staff should be realistic, and that it is possible to make the best use of existing staff without requiring an abundance of funding, expensive outside vendors or consultants, or a dedicated project. 


\section{Scenarios}

The following five scenarios articulate typical approaches to preservation of audiovisual materials, providing hypothetical conditions that influence decisions about what preservation actions to take. They focus on a broad range of typical and recommended implementations based on this report, and summarize the above knowledge and the different ways it can be implemented given specific situations.

\subsection{Academic Archives}

Fonds University is a top-level research institution with strong Bioengineering, Computer Science, and Digital Media departments. Their Archives and Special Collections department aggressively collects work from prominent alumni, including recent digital archives. The University Library's archives department consists of 30 librarians and archivists and 30 IT staff dedicated to processing material through their in-house preservation system. It is crucial that all their audiovisual materials from dozens of collections and sources are treated alongside other digital data in their repository and seamlessly accessed through their frontend user portal. Their audiovisual material has a wide range: digital artworks, videos taken from laptop images, oral histories recorded on DAT tapes, Umatic videotapes from the campus television network, and more. When given a choice during the act of digitization, archivists choose to preserve audio at $96 \mathrm{kHz}$ resolution and 24 bits per sample, the standard for audio preservation. For video, archivists choose FFV1 codec with LPCM audio wrapped in a Matroska container, due to the robust tooling available during the capture, transfer, and quality control stages of preservation. Because Fonds University Archives do their digitization work in house with a staff of 12 technician staff, they are able to make decisions around what is the best choice for the overall preservation budget and for their customized workflows. Access copies are generated as $\mathrm{mp} 3$ for audio and h264-encoded $\mathrm{mp} 4$ for video, due to compliance with web performance guidelines and maximum cross-compatibility. Their access files are generated in three different qualities $-92 k, 160$, and 320k for the audio access copies, and varying degrees of h264 compression for the video access copies.

\subsection{Broadcast Archives}

TV4ME is a medium-sized broadcast television station. Their archives actively collect all broadcast material running on three channels, 24 hours a day. This full-scale operation has an automated system that pulls data from the live video streams and writes them directly into a digital asset management system. It does not seek to retain all data, but only original programming. Syndicated media is not captured and kept, and this cuts down on storage costs and legal issues. This automated process also replicates copies and moves them to geographically distinct locations, stored on tape. The television station does not just seek to archive its live television, but also the associated audio and video recordings that create the original programming. This material is processed by a team of technicians, and the media ingested into their digital asset management system. For an organization with a very large throughput, it is essential that the process be fast, automated, and completely sturdy. TV4ME has a full team working around the clock to ensure the process continues to function. Backups are available on tape, which means they are not available instantly, but can be accessed anywhere from within a few minutes up to a few hours if necessary. It makes sense to compromise and optimize for speed at the point of ingest into the repository, even if it means taking a little longer with retrieval of archival material.

\subsection{Contemporary Art Museum}

Artlife Museum, a private contemporary art museum, acquired an immersive $360^{\circ}$ virtual reality artwork that relies on a VR headset, a consumer-level drone, and a bowl of mayonnaise. The 
museum's conservators are interested in preparing the dataset they received from the artist for an upcoming exhibition, but are also proactively preparing the content for preservation storage and to be able to loan out the artwork or re-exhibit it in the future. To begin the acquisition process, they interview the artist repeatedly, getting as many details about the piece and the intent behind it, and document her answers. They record the model number of the preferred VR headset and drone, and note the brand of mayonnaise and type of bowl. After months of research, sufficient detail is collected about the piece including guidelines around presentation and interviewing the artist to get any other insight, and the digital components are considered ready for preservation. The software for this presentation is specific to the associated VR equipment and is in a proprietary format. The preservationists gather as much technical metadata about the software as possible through research into the files and operating system, including research into how the artist was able to create the experience. Then, the digital components are processed through a preservation processing system, creating an AIP out of the project and attaching related documentation on the additional components. No additional versions of the software are created for access or preservation purposes, except duplicated copies stored across three geographical locations managed by Artlife Museum staff. While in storage, checksum monitoring is a constant, ongoing process to ensure bits remain exact as intended until a copy of the software is ready for retrieval again in the future.

\subsection{Small Historical Society}

Mediumsville Historical Society, a small municipal archives organization focused on local history, received a small grant to digitize and preserve a dozen small-gauge films featuring home movies from a local family, dating from the late 1940s to late 1950s. Staff time and expertise are both limited. Staffing consists of a director, two full-time staff with multiple job responsibilities, and two part-time processing archivists. The grant budget only covers the cost of digitization, not preservation storage or a framework necessary for providing web-based access. The Historical Society ships materials to a reputable film digitization vendor, known for working with the cultural heritage sector and for the purpose of preservation. A month or so later, the films are returned with a hard drive containing two copies of each film. One very large file does not replay on the Historical Society's older computers but is functional on a personal laptop belonging to one of the processing archivists. The other file is smaller, and is playable on their work machines. The Historical Society was gifted the home movies and their donor form assigns copyright to them. For the preservation primary copy, they copy the files onto two replicated drives and store this with the rest of their digital collections. One is stored in an off-site location where the material is also replicated multiple times. The Historical Society ingests the access copies of the films into their digital collections website, powered by Omeka, which holds their other digitized image and document materials.

\subsection{Community Archives}

Treehouse Community Archives is an informal archive for an intentional cohousing community, a cooperative neighbourhood focused on sharing responsibilities and space, with monthly meetings and weekly shared dinners. The group, formed in 1970, resides in a cluster of homes with a large common area. They also run a small farm and shares these resources. The archive consists of business documents, photographs, news clippings, T-shirts, and small-gauge film reels and videotapes from events and monthly general meetings, where decisions are made. The group is preparing to celebrate their 50th anniversary, and need to gather material from the archives for a small documentary being put together by a local filmmaker. One group member has solicited her daughter, a trained archivist, to manage the organization and digitization of audiovisual elements for the filmmakers to use, and has gathered volunteers from the community to do the work. The archivist outlines a plan to get materials stored in the most stable environment possible, which is a 
cupboard in the community centre. Materials are sorted and videotapes are digitized by community members using a basic VHS deck and a consumer-grade analogue-to-digital converter, to the highest possible quality available but still a lossy format. Film materials of interest are sent to a local vendor to be digitized, with funding provided by the local filmmaker. When the materials return, their digital surrogates are combined with the other materials and stored on a small RAID drive at the community centre. The collective votes not to send data to outside sources, so additional copies are made and stored within the community. They accept that this is a risk. They draft a plan to regularly check and migrate the material to new hardware, every three years, and plan to research and invest in an additional storage method. While this is not the ideal preservation solution, it is the best possible solution for a small institution with no technical staff or budget. Clips that do not contain sensitive information are uploaded to the Internet Archive, and community members are able to share the videos on social media and with their friends and family. 


\section{Conclusion}

This report began by covering some essential concepts associated with analogue and video material, starting with how to categorize and understand the three major types of audiovisual material: analogue, digital, and hybrid formats. This should give readers a head-start on assessing their audiovisual collections in anticipation of taking preservation action. Following this summary of format types was a deeper dive into digital audiovisual files and some of their essential technical characteristics. Having this foundation of digital video will support the active practice of preserving and making digital archival objects accessible. It should be helpful as a guide for when preservationists run into specific problems associated with the technical components of audio and video files.

When working with files, the Practical Best Practices section should serve as a guide for how to get started in these endeavours. Remember that the recommendations are meant to be flexible, and adapted to the unique situation that each organization doing preservation work finds itself in. Following Practical Best Practices is a look at issues that arise when carrying out preservation work with audiovisual materials for the first time, which will serve to highlight things to be aware of when planning projects.

The Case Studies and Scenarios sections should provide inspiration for how to customize the Practical Best Practices to work for specific situations and institutional priorities. They focus on the different ways that an organization may address the handling of this type of material, depending on their allocated priorities, time and finances.

In summary, it is important to remember that preservation is an ongoing process and preservationists should seek to do the best they can with what they have available to them. Imperfect action is better than no action at all! Especially for analogue moving image and sound materials, there is very little time to wait before capturing the material becomes impossible. 


\section{Glossary/Terminology}

Acetate: a film base created to replace the more dangerous nitrate, used in major motion picture film as well as small-gauge film. When aging in poor conditions, it can suffer from 'vinegar syndrome', named after its smell.

Bottom Field First (BFF): a way to determine the order of interlaced frames.

Burnt-In Time Code (BITC): data that is stored as part of the image frame. This cannot be removed.

Command line: a text-only interface for interacting with a computer.

Compressed: data that has been made smaller in some way.

Display Aspect Ratio (DAR): the combination (which occurs by multiplication) of both the pixel aspect ratio and storage aspect ratio giving the aspect ratio as experienced by the viewer.

Line 21: captions that follow the EIA-608 standard, referred to by the field the captions operate on. Linear Timecode (LTC): data stored as a separate audio track.

Lossy compressed: data has been made smaller and this cannot be undone.

Losslessly compressed: data has been made smaller in some way, but the process is reversible.

Nitrate: also referred to as cellulose nitrate or nitrocellulose, this format was used at the advent of the film age in the 1880s up until the 1950s. While it is not dangerous to handle, it is much more flammable than other film bases and there may be local regulations that require the film to be stored and transported carefully.

Pixel Aspect Ratio (PAR): the horizontal to vertical ratio of each, rectangular, physical pixel.

Polyester: the most modern film base, and often used when creating preservation-grade copies of films on film due to its stability.

PREMIS: an acronym for PREservation Metadata: Implementation Strategies, a digital preservation metadata standard

RGB: Red, Green, Blue, an additive form of colour space.

Storage Aspect Ratio (SAR): the horizontal to vertical ratio of solely the number of pixels in each direction.

Spinning disk: electro-mechanical storage consisting of a hard drive that uses magnetic storage to retrieve digital data.

Top Field First (TFF): a way to determine the order of interlaced frames.

Uncompressed: data stream with no attempts to decrease size.

Vertical Interval Time Code (VITC): data is stored in the 'vertical blanking interval' of a video track. This means the data is stored as part of the video stream, on a single non-visible scan line.

YCbCr: $\mathrm{Y}$ represents the luma, and $\mathrm{Cb}$ and $\mathrm{Cr}$ represent the blue-difference and red-difference of an image.

YUV: $Y$ represents the luma, and $U$ and $V$ represent colour data in an image. 


\section{Further Reading and Resources}

Brylawski , S., Lerman, M., Pike, R., Smith, K., editors. The ARSC Guide to Audio Preservation Available at: https://web.archive.org/web/20200927033909/https://www.clir.org/wp-

content/uploads/sites/6/pub164.pdf last accessed 23/09/2020

Cocciolo, A. 2017. Moving Image and Sound Collections for Archivists. Chicago: Society of American Archivists.

Gracy, K. 2007. Film Preservation: Competing Definitions of Value, Use, and Practice. Chicago: Society of American Archivists.

IASA 2009 Guidelines on the Production and Preservation of Digital Audio Objects (web edition) https://web.archive.org/web/20201001081959/https://www.iasa-web.org/tc04/audio-preservation last accessed 23/09/2020

Jarczyk, A., Kromer, R., and Pfluger, D. 2020. 'Memoriav Recommendations: Digital Archiving of Film and Video: Principles and Guidance', Version 1.2. Available at:

https://web.archive.org/web/20201001082130/https://memoriav.ch/dafv/?lang=en last accessed $23 / 09 / 2020$

Jimenez, M., Platt, L. Texas Commission on the Arts 2004. 'Videotape Identification and Assessment Guide' Available at: https://web.archive.org/web/20201001082255/https://www.arts.texas.gov/wpcontent/uploads/2012/04/video.pdf last accessed 23/09/2020

Morel, M, Bay Area Video Coalition 2019. 'A Guide to Approaching Audiovisual Digitization for Artists and Arts and Culture Organizations' Available at:

https://web.archive.org/web/20190709030023/https://bavc.org/sites/default/files/wysiwyguploaded/BAVC Guide To Audiovisual Preservation 2019 0.pdf last accessed 23/09/2020

Poynton, C. 2003. A Technical Introduction to Digital Video. New York: J. Wiley.

\section{Educational resources}

Nederlands Instituut voor Beeld en Geluid Winter School: A regularly held training programme for audiovisual archivists featuring experts in the field. More information available at:

https://web.archive.org/web/20200707090140/https://www.beeldengeluid.nl/en/knowledge/winte r-school-audiovisual-archiving last accessed 23/09/2020

'No Time to Wait!' Symposium: An annual conference connecting open-source software developers, standards authors, and preservationists with a focus on audiovisual preservation More information available at: https://web.archive.org/web/20200824174613/https://mediaarea.net/NoTimeToWait last accessed 23/09/2020

open-workflows: A regularly updated page of references to workflows, publications, and tools associated with audiovisual preservation. Available at:

https://web.archive.org/web/20201001084530/https://github.com/amiaopensource/openworkflows last accessed 23/09/2020

Sustainable Heritage Network: A resource providing 'comprehensive workshops, online tutorials, and web resources dedicated to the lifecycle of digital stewardship'. More information available at: https://web.archive.org/web/20201001084827/https://www.sustainableheritagenetwork.org/ last accessed 23/09/2020 


\section{References}

Casey, M. 2015. 'Why Media Preservation Can't Wait: The Gathering Storm' IASA Journal 44, pp. 1422. Available at: https://web.archive.org/web/20200906155506/https://mdpi.iu.edu/doc/storm.pdf last accessed 23/09/2020

CCSDS Secretariat. 'Reference Model For An Open Archival Information System (OAIS)' (PDF). June 2012. Available at:

https://web.archive.org/web/20200917202243/https://public.ccsds.org/Pubs/650x0m2.pdf last accessed 30/9/2020

Dance/USA. 2020 'Artist's Legacy Toolkit: Copyright'. Available at:

https://web.archive.org/web/20200906155409/https://www.danceusa.org/archiving-preservationartists-legacy-toolkit-copyright last accessed 23/09/2020

Digital Preservation Coalition 2019. 'The 'Bit List' of Digitally Endangered Species' Available at: https://web.archive.org/web/20200906155338/https://www.dpconline.org/digipres/championdigital-preservation/bit-list last accessed 23/09/2020

Documenting the Now. Available at:

https://web.archive.org/web/20200819122339/https://www.docnow.io/ last accessed 23/09/2020

Duryee, Alex 2014. 'An Introduction to Optical Media Preservation'. Code4lib Journal 24. Available at: https://web.archive.org/web/20200724200331/https://journal.code4lib.org/articles/9581 last accessed 23/09/2020

Jarczyk, A., Kromer, R., and Pfluger, D. (2020). 'Memoriav Recommendations: Digital Archiving of Film and Video: Principles and Guidance', Version 1.2, pp. 49-51. Available at:

https://web.archive.org/web/20190815000000*/https://memoriav.ch/dafv/?lang=en last accessed $\underline{23 / 09 / 2020}$

Library of Congress 2020. Sustainability of Digital Formats: Planning for Library of Congress

Collections: Material Exchange Formats. Available at:

https://web.archive.org/web/20200810000242/https://www.loc.gov/preservation/digital/formats/f

$\mathrm{dd} /$ fdd000013.shtml last accessed 23/09/2020

MIPoPS 2020. DVRescue. Available at:

https://web.archive.org/web/20200930100348/https://www.mipops.org/dvrescue/ last accessed $23 / 09 / 2020$

PREFORMA 2018. 'Preforma Project'. Available at:

https://web.archive.org/web/20200717174536/http://www.preforma-project.eu/ last accessed $23 / 09 / 2020$

Project Arcc. Available at: https://web.archive.org/web/20200414203221/https://projectarcc.org last accessed 23/09/2020

Rice, Dave 2015. 'Sustaining Consistent Video Presentation'. Tate. Available at:

https://web.archive.org/web/20191229003033/https://www.tate.org.uk/about-

us/projects/pericles/sustaining-consistent-video-presentation last accessed 23/09/2020 
Toth, B 2019. 'Knowledge Obsolescence in Audiovisual Preservation: Legacy Skills as Cultural Heritage' Available at: https://web.archive.org/web/20200621175803/https://www.briannatoth.com/research last accessed 23/09/2020

Wikipedia, Advanced Video Coding: Profiles 2020. Available at:

https://web.archive.org/web/20200904094507/https://en.wikipedia.org/wiki/Advanced Video Cod ing last accessed 23/09/2020

WITNESS 2020. Available at:

https://web.archive.org/web/20200903143046/https://www.witness.org/ last accessed 23/09/2020

Wright, R 2012. Technology Watch Report: Preserving Moving Pictures and Sound. York: Digital Preservation Coalition. Available at: https://www.dpconline.org/docs/technology-watchreports/753-dpctw12-01-pdf/file last accessed 23/09/2020

\section{Tools referenced}

FFmpeg 2020. Available at:

https://web.archive.org/web/20200823185002/https://www.ffmpeg.org/ last accessed 23/09/2020

MediaConch 2020. Available at:

https://web.archive.org/web/20200810055951/https://mediaarea.net/MediaConch last accessed $23 / 09 / 2020$

RAWcooked 2020. Available at:

https://web.archive.org/web/20200816002246/https://mediaarea.net/RAWcooked last accessed $23 / 09 / 2020$

vrecord 2020. Available at:

https://web.archive.org/web/20200626094818/https://github.com/amiaopensource/vrecord last accessed 23/09/2020

Wayback 2020. Available at:

https://web.archive.org/web/20200811061624/https://github.com/internetarchive/wayback/ last accessed 23/09/2020

Webrecorder 2020. Available at:

https://web.archive.org/web/20200830194722/https://webrecorder.net/ last accessed 23/09/2020 